\title{
1 Precision environmental health monitoring by longitudinal exposome and
}

\section{2 multi-omics profiling}

3 Peng Gao ${ }^{1,3}$, Xiaotao Shen ${ }^{1,3}$, Xinyue Zhang ${ }^{1}$, Chao Jiang ${ }^{2}$, Sai Zhang ${ }^{1}$, Xin Zhou ${ }^{1}$, Sophia Miryam

4 Schüssler-Fiorenza Rose ${ }^{1}$, Michael Snyder ${ }^{1, *}$

$5 \quad{ }^{1}$ Department of Genetics, Stanford University School of Medicine, Stanford, CA, USA

$6 \quad{ }^{2}$ Life Sciences Institute, Zhejiang University, Hangzhou, Zhejiang, China

$7 \quad{ }^{3}$ These authors contributed equally

$8 \quad$ *Corresponding email: mpsnyder@stanford.edu

\section{Abstract}

11 Conventional environmental health studies primarily focused on limited environmental stressors at the

12 population level, which lacks the power to dissect the complexity and heterogeneity of individualized

13 environmental exposures. Here we integrated deep-profiled longitudinal personal exposome and internal

14 multi-omics to systematically investigate how the exposome shapes an individual's phenome. We

15 annotated thousands of chemical and biological components in the personal exposome cloud and found

16 they were significantly correlated with thousands of internal biomolecules, which was further cross

17 validated using corresponding clinical data. In particular, our results showed that agrochemicals (e.g.,

18 carcinogenic pesticides, fungicides, and herbicides) and fungi predominated in the highly diverse and

19 dynamic personal exposome, and the biomolecules and pathways related to the individual's immune

20 system, kidneys, and liver were highly correlated with the personal external exposome. Overall, our

21 findings demonstrate dynamic interactions between the personal exposome and internal multi-omics and

22 provide important insights into the impact of the environmental exposome on precision health. 


\section{Introduction}

24 Human health is shaped by the personal genome, microbiome, and exposome [1]. Extensive studies have

25 been conducted on the genome and microbiome, however, the human exposome is rarely investigated,

26 especially at the individual level. Exposomics research aims to characterize all physical, chemical, and

27 biological components collectively in the human external and internal environment. The external

28 environment consists of all potential exposures from the near-field to the far-field sources of exogenous

29 chemical, biological, and physical exposures [2-5]. The internal environment includes but is not limited to

30 dietary components [6-8], xenobiotics and their biotransformation products, foreign DNA/RNA, and

31 bioactive molecules accumulated from exogenous sources [9].

33 Conventional environmental health risk assessments rely on environmental epidemiology within a

34 predefined, usually large, geographical region. However, recent studies revealed that personal exposome

35 profiles are highly dynamic and spatiotemporally different among individuals who live in the same

36 geographical area. For instance, studies have shown that individuals are exposed to significantly different

37 chemical and biological stressors during the same period even if they are in the same general geographical

38 region, such as the San Francisco Bay Area or London [10,11]. Another limitation is that previous studies

39 usually targeted a single group of stressors, which failed to provide a holistic picture of the exposome cloud

40 and their interactions [12]. Moreover, stressor-induced physiological responses varied significantly among

41 different individuals [11]. Therefore, there is a critical need to monitor exposures at the individual level and

42 systematically integrate them with respective internal multi-omics profiles to fully characterize each

43 individual's personal responses to environmental exposures.

45 Multi-omics analyses enable a detailed investigation into the biological mechanisms underlying human

46 phenotypes by integrating multiple omics, such as proteomics, metabolomics, and microbiomics [13].

47 Multi-omics profiling, together with clinical measures such as cytokines and blood tests, can

48 comprehensively assess one's health status and detect significantly correlated exposures to understand the 
impact of the external exposome on human biology and health [10,14,15]. In addition, longitudinal profiling can avoid biases introduced by one-time sampling and provide a molecular portrait of the effect of different

51 exposures at an individual level.

53 In this first of its kind study, we utilized our previously published datasets to integrate thousands of

54 longitudinally measured chemical and biological components along with physical factors in the personal

55 exposome to investigate how the various stressors in the external exposome impacted internal -omes, such

56 as the proteome, metabolome, the gut microbiome as well as cytokines and blood markers $[10,14,15]$.

57 Specifically, this study 1) improved the annotation of biological and chemical exposures in the external

58 exposome and human blood; 2) integrated the external exposome with internal multi-omics to investigate

59 the effect of the exposome on molecular phenotypes and pathways; and 3) correlated the environmental

60 stressors with clinical measurements to associate the health effects of the external exposome. Overall, we

61 found thousands of external chemical and biological exposures associated with the internal microbial,

62 proteomic, and metabolic alterations indicating a strong correlation between the external exposome and

63 molecular health.

65 Results

66 Longitudinal profiling of the exposome and internal multi-omics to monitor personal environmental

67 health

68 We investigated whether the external exposome is related to the internal molecular and physiology profile

69 at a comprehensive and personal level using the schematic shown in Figure 1a. We first reanalyzed deep

70 biological and chemical exposome data collected from our previously published study in which an

71 individual had been continuously wearing a personal exposome collection device "exposometer" and

72 correlated it with the internal molecular profiles. Over the 52-day period relevant for this study, the device

73 captured organic chemicals using zeolite, followed by methanol elution and liquid chromatography coupled

74 to high-resolution mass spectrometry (LC-HRMS) analysis. Biological specimens were also captured using 
75 polyethersulfone filters and nucleic acids analyzed by high throughput sequencing of DNA and RNA.

76 (Supplementary Data 1). General environmental factors (e.g., temperature, humidity, total particulate

77 matter) were recorded by the device, and the other environmental factors were also obtained from the local

78 air quality monitoring stations (Figure 1a). Contrary to conventional exposome monitoring studies, which

79 usually focused on the exposures at a single timepoint [16-18], we captured personal exposome profiles

80 across 18 timepoints, and annotated 1,265 genera, 158 known chemical stressors among 3,299 chemical

81 features, and 10 environmental factors which include physical stressors that may impact environmental

82 health in this study (Figure 1b). These genera and known chemical stressors were annotated from improved

83 microbiome and chemical annotation pipelines that we developed as part of this study (Methods).

85 Over the same 52 days period, we also collected stool and blood samples from the same participant to 86 profile the gut microbiome, proteome, metabolome, toxins and carcinogens, cytokines, and blood tests

87 (Figure 1c and Supplementary Data 1). Through reanalysis pipelines, we were able to annotate 60 toxins 88 and carcinogens as well as 664 metabolites, 302 proteins, and 62 gut microbiome taxa. We also measured 8962 cytokines and 46 clinical blood parameters to longitudinally monitor personal health status [15]. All 90 sample collections were performed during the first quarter of 2016 from three distinct locations in the U.S.

91 (Figure S1). However, not all sample types were collected at each time point, and the inter-omics analyses

92 were performed only when overlaps were available (Figure 1b). Despite our limited ability to control all 93 confounding variables, we searched for significant intra- and inter- exposome correlations and high-degree 94 components which have the most significant correlations in each analysis as those may play important roles 95 in the exposome-ome interactions $[|r|>0.9$; False Discovery Rate (FDR) adjusted p-value (q-value) $<0.05]$.

\section{Intra-exposome relationships in the highly dynamic and diverse personal exposome cloud}

97 To annotate as many chemicals as possible, we searched through the 3,299 LC-HRMS raw features using

98 a combination of five public exposome related databases as well as an in-house database that we assembled.

99 Using this new annotation pipeline, we were able to annotate 158 known chemical stressors (Figure 2a,

100 Methods). These stressors were categorized into 13 classes, with the dominant class being agrochemicals, 
101 followed by pharmaceuticals \& personal care products (PPCPs), plasticizers, and International Agency for

102 Research on Cancer (IARC) Group 2A carcinogens, and the chemicals in each class varied dynamically

103 during the monitoring period (Figure 2a, Figure S2 and Supplementary Data 2). To characterize the

104 biological exposome domain, we circumvented the limited ability of $16 \mathrm{~S}$ rRNA/18rRNA/ITS sequencing

105 by applying metagenomic sequencing. We found 17 genera dominated during the study period, most of

106 which were fungi and bacteria, but they varied dynamically (Figure 2b). Ten general environmental factors,

107 measured either by personal exposometer (temperature, humidity, and total particulate matter) or local air

108 monitoring stations (atmospheric pressure, wind speed, $\mathrm{SO}_{2}, \mathrm{NO}_{2}, \mathrm{O}_{3}, \mathrm{CO}$, and air quality index), were also

109 included in the study (Figure 2c).

111 We performed intra-omics correlation analyses to investigate the potential relationships among all the

112 exposome components (Methods, Supplementary Data 3). We found a total of 60 statistically significant

113 correlations $(|r|>0.9$ and q-value < 0.05) among 74 exposome components, including 41 chemicals, 30

114 genera, and 3 environmental factors (Figure 2d and Figure S3). Specifically, diisononyl phthalate (a

115 plasticizer) and butylated triphenyl (an organophosphate flame retardant) had the most significant

116 correlations, followed by various agrochemicals, PPCPs, and IARC group 2A carcinogen. Among the

117 biological components, Tricholoma had the highest number of significant correlations, followed by

118 Cylindrobasidium, Piriformospora, Erysiphe, Schizophyllum, Serendipita, and Hirsutella, all of which are

119 fungi (Figure 2e). In terms of environmental factors, only temperature and humidity collected by the

120 exposometer as well as $\mathrm{SO}_{2}$ concentration collected by the local monitoring stations were significantly

121 correlated with other exposome components (Figure 2d). For example, Paenibacillus was positively

122 correlated with the temperature, consistent with the literature that members of Paenibacillus are heat

123 resistant and grow well in relative hot temperatures [19]. Azoxystrobin, ethylparaben and captan are

124 fungicides or antifungal agents [20,21] that negatively correlated with different fungi (Figure 2f). 
126 Inter-omics analyses between the exposome and multi-omics revealed physiological links to the

127 exposome

128 To investigate how the exposome shapes an individual's phenome longitudinally, we investigated the links

129 between the exposome and internal multi-omics. Specifically, we found 8,986 significant correlations $(|\mathrm{r}|>$

$130 \quad 0.9$ and q-value < 0.05) among 1,700 factors from all -omes, and positive correlations were more

131 predominant than negative correlations (Figure 3a, Supplementary Data 4). The biological exposome and

132 metabolome were the most extensive -omes in the network, and they also had the greatest number of

133 significant correlations $(\mathrm{N}=4148$; Figures 3a and 3b). Additionally, we found that the exposome and

134 internal multi-omics networks can be divided into several subnetworks with high modularity (0.819, Figure

135 S4a, b).

Personal exposome-gut microbiome interactions

138 We found 1,333 significant correlations $(|r|>0.9$ and q-value $<0.05)$ between the exposome and the gut

139 microbiome (16S rDNA data), and the number of positive and negative correlations were approximately

140 equal (Figure 3c). Specifically, the six highest-degree bacteria (each correlates with 34 exposome

141 components) may be involved in multiple physiological processes that respond to the personal exposome.

142 For example, members from Alistipes were shown to play essential roles in inflammation and various

143 diseases [22], members from Eggerthella were implicated as the causes of liver and anal abscesses,

144 ulcerative colitis, and systemic bacteremia [23], members from Odoribacter were found to maintain short-

145 chain fatty acid availability and systolic blood pressure [24], members from Parasutterella were involved

146 in bile acid maintenance and cholesterol metabolism [25], whereas members from Roseburia played vital

147 roles in producing short-chain fatty acids and anti-inflammatory pathways [26]. Out of the top six genera,

148 all but Roseburia positively correlated with chemical stressors and usually negatively correlated with

149 biological components (Figure 3d and S4d). As a result, members from Alistipes, Eggerthella,

150 Odoribacter, and Parasutterella were more likely to be involved in proinflammatory processes, while

151 members from Roseburia were mainly involved in anti-inflammatory processes. On the exposome side, 
153 gut bacteria) among all exposome components, indicating their essential roles in interacting with the

154 participant's gut microbiome (Figure 3c). Overall, these results demonstrate an association of the external 155 exposome with the gut microbiome and its associated biological processes, particularly inflammation.

\section{Exposome-proteome interaction network}

158 We found 2,054 statistically significant correlations $(|\mathrm{r}|>0.9$ and q-value $<0.05)$ between the individual's 159 exposome and internal proteome. Most of the high-degree exposome components were biological 160 components, and positive correlations were slightly more frequent than negative correlations (Figure 4a).

161 Specifically, we found 11 highest-degree substances (nine genera and two chemicals), each of which was 162 significantly correlated with more than 22 proteins in the proteome. The high-degree biological genera were 163 fungi and primarily positively correlated with proteins; in contrast, Xeromyces negatively correlated with 164 proteins. Fenazaquin (a pesticide) and tetrabromobisphenol A diallyl ether (a brominated flame retardant) were two high-degree chemical stressors, both of which primarily negatively correlated with proteins. On the proteome side, 17 highest-degree proteins (each correlated with 21 exposome components) were

167 discovered, and 14 of these are directly immune-related. For instance, alpha-1-HS-glycoprotein (AHSG) 168 promotes endocytosis, complement component 3 (C3) activates the complement system, and fibrinogen 169 alpha chain (FGA) is involved in both innate and T-cell mediated pathways [27]. Additionally, we 170 discovered significantly correlated signaling pathways when queried against GO, KEGG, and Reactome 171 databases (Supplementary Data 5 and 6). Chemical and biological exposome shared several significantly 172 correlated pathways, such as protein activation cascade, platelet degranulation and acute inflammatory 173 response, whereas some pathways were uniquely correlated with the chemical exposome, such as platelet 174 activation, signaling, and aggregation pathway (Figure 4b). Moreover, immune-related pathways were 175 among the most common high-degree signaling pathways correlating with chemical and biological 176 exposome, and those pathways were about half positively and half negatively correlated with the exposome

\section{7 (Figure 4c).}




\section{Exposome-metabolome interaction network}

180 The blood metabolome is considered the most interactive -ome with the exposome since xenobiotics

181 interact with endogenous metabolites initially after entering the human body. In fact, the blood exposome

182 overlaps with the blood metabolome from an analytical perspective as current approaches cannot

183 distinguish the sources of the molecules present in blood. Moreover, xenobiotic biotransformation is similar

184 to that of metabolic pathways and can even involve the same enzymes, such as cytochromes P450 [16,28].

185 Therefore, it is essential to investigate the interactions between the exposome and metabolome to better 186 understand the initial health impact of the exposome.

188 In this study, we found 4,624 statistically significant correlations $(|\mathrm{r}|>0.9$ and q-value $<0.05)$ between the 189 exposome and internal metabolome. Specifically, positive correlations were more frequent in the 190 exposome-metabolome analysis than the exposome-proteome analysis (Figure 5a and S5). The high191 degree biological components were primarily fungi and usually positively correlated with the metabolites;

192 interesting exceptions are Aegilops (a grass), the bacteria Pontibacter and Hymenobacter, and Paramecium

193 (a ciliated protist). Salicylic acid (a PPCP), dinoseb (an herbicide), dibromoethane (an IARC group 2A 194 carcinogen) were the three highest-degree chemicals, all of which primarily positively correlated with 195 endogenous metabolites. Importantly, we found 19 high-degree metabolites, each significantly correlated 196 with 21 exposome substances. Several metabolic pathways were significantly correlated with both the 197 chemical and biological exposome (Methods, Figure S7), such as protein digestion and absorption and 198 aminoacyl-tRNA biosynthesis, whereas some pathways were only correlated with the biological exposome

199 (Figure 5b, Supplementary Data 7 and 8). Similar to the exposome-proteome analysis, we performed 200 correlation network analysis among the exposome, metabolites, and metabolic pathways. Trimethyl 201 phosphate (a plasticizer and organophosphate flame retardant), 2,2',3,3',4,4',5-Heptachlorobiphenyl (a 202 polychlorinated biphenyl), and tetrachloroethylene (an IARC group 2A carcinogen) were positively 
correlated with all the metabolic pathways, whereas tetrabromobisphenol A diallyl ether, salicylic acid, and zeranol (a mycotoxin) were negatively correlated with all metabolic pathways (Figure 5c).

\section{Monitoring precision environmental health by investigating the exposome-clinical data correlations}

207 Standard clinical measurements such as blood and cytokine tests directly reflect the individual's health.

208 Thus, clinical test results are ideal indicators to investigate the health impact of the exposome. Based on

209 our exposome-cytokine analysis, the biological exposome had the most significant correlations with

210 cytokines, followed by chemical and environmental factors. 362 significant correlations $(|\mathrm{r}|>0.9$ and q-

211 value $<0.05$ ) were found between the exposome and cytokines, most of which were positive correlations.

212 After converting correlation coefficients to variable importance in projection scores, we determined the

213 contributions of all significantly correlated exposome components on cytokines (Supplementary Data 10,

214 Methods). Specifically, 60\% of the cytokine variation was explained by the determined factors in this

215 study. Furthermore, the top 13 cytokines, which were almost entirely contributed by the annotated

216 exposome components (> 90\%), were all proinflammatory cytokines, such as IL-23, MCP-1, and VCAM-

217 1, indicating that those cytokines may play essential roles in response to the exposome. Additionally, 14

218 highest-degree (each correlates with > 7 exposome components) cytokines were found to be primarily

219 positively correlated with the exposome, whereas only MCP-1 was primarily negatively correlated (Figure

220 6a). The most high-degree biological components were fungi, such as Wallemia, which, interestingly, are

221 filamentous food-borne pathogens [29]. Moreover, other than Xeromyces, most of the exposome

222 components were primarily positively correlated with cytokines, consistent with the exposome-proteome

223 analysis where Xeromyces primarily negatively correlated the proteins. Interestingly, acephate (an

224 insecticide) is the highest-degree chemical component, positively correlated with 10 cytokines (Figure 6a).

226 Similar to the exposome-cytokine analysis, biological components had the most significant correlation with

227 blood tests, followed by chemicals and environmental factors. However, fewer chemicals were correlated

228 with blood tests than those correlated with cytokines (Figure 6a). 513 significant correlations were found 
229 between the exposome and blood tests, and the majority were positive correlations. Using similar 230 contribution determination algorithms, $77 \%$ of the blood tests variation was explained by the determined 231 factors in this study. Similarly, the top 13 blood tests which were almost entirely contributed by the 232 determined exposome components (contributions of the exposome $>95 \%$ ), were primarily related to the 233 immune system, liver, and kidney functions. Additionally, eight highest-degree (each correlated with > 25

234 exposome components) blood tests were primarily positively correlated with the exposome while only 235 platelet was primarily negatively correlated. Interestingly, the highest-degree blood test, creatinine, which 236 is a biomarker for kidney function, correlated with 62 exposome components (Figure 6b). Unlike cytokine 237 profiles, where we cannot draw a clear line of the individual's health status, blood tests have clinically 238 established reference ranges facilitating the clinical impacts. We therefore performed correlation analyses 239 to understand the effects of the exposome on personal health using blood test results with out-of-range 240 values. Interestingly, we found the abnormal blood glucose level values were significantly correlated with 2413 chemical stressors and 13 microbes. For instance, salicylic acid concentration was negatively correlated 242 with glucose level; salicylic acid has been previously shown to decrease glucose concentration and used as 243 a treatment for type 2 diabetic patients [30], which is consistent with our findings. Similarly, abnormal 244 values of absolute eosinophils and urea nitrogen correlated with specific biological exposome components, 245 including some known pathogens, such as Aureobasidium, Niastella, and Scedosporium (Figure 6b). 246 Previous studies were consistent with our results as eosinophilic phagocytosis consumes eosinophils during 247 allergy and inflammation [31], and various pathogenic microbes can utilize urea as a nitrogen source [32].

\section{Discussion}

250 It has long been acknowledged that environmental factors affect personal health, but conventional 251 environmental health studies face limitations. For example, a) population or cohort studies overlook the 252 significant differences between individuals; b) single timepoint sampling fails to reflect the continuous 253 effects of stressors; c) and focusing on a single or a class of stressors does not capture the holistic health 254 impact of the exposome. To overcome these challenges, we generated a comprehensive precision 
environmental health profile by longitudinally monitoring both the personal exposome and internal multieffects of the exposome. Using Spearman correlation analysis, we discovered many significant correlated physiological parameters and exposome components, indicating their interactions in the participant's responses to the personal exposome. Additionally, our study provided vast testable hypotheses to further investigate the underlying mechanisms using analytical and experimental approaches.

We were able to capture more than chemical 3,000 features, but only annotated 158 known chemical stressors by a broad annotation method that utilizes various databases, including those containing emerging contaminants [33-36]. This indicates that existing exposome databases still lack the power to annotate the majority of the chemical exposome. Interestingly, we found that the concentrations of most chemicals increased after January 25, 2016, when the individual transitioned from a period of residing at home to a period of high travel, indicating that the chemical exposome greatly increased with travel to other locations studied chemical stressors, making them most easily identifiable. It is also worth noting that high

271 concentrations do not necessarily imply high health risks since each chemical has its own safe dose, and

272 the combined effects among them are still unclear [13].

274 The biological exposome revealed a number of interesting observations as well. The fungal genus Stereum 275 was dominant at most time points, reflecting its high abundance in the personal exposome (Figure 2a). We 276 found several interesting correlations of the biological exposome with chemical and environmental factors, 277 such as associations of antifungals with a decrease in fungal exposures. Many of these are intended food or 278 soil antifungal products (azoxystrobin and captan), whereas others are common preservatives (ethylparaben 279 and anthracene) that have antifungal properties [10,11]. Overall, nearly 100 significant correlations were 280 found by intra-exposome analysis, representing the complex interactions within the exposome domains. 
281 Importantly, the negative correlations of fungi with various pesticides and herbicides indicate these

282 agrochemicals may inhibit the fungi growth as well (e.g., Tricholoma versus propoxur and Erysiphe versus

283 bentazone). Moreover, we find several interesting tertiary relationships, such as the mycotoxin fusarin C

284 (produced by Fusarium) negatively correlated with Cylindrobasidium, suggesting a possible competition

285 among the different fungi (Supplementary Data 3).

287 A recent study identified radioprotective gut microbes and internal metabolites in mice using a multi-omics

288 analysis [37], demonstrating the potential of this approach to investigate essential components in the

289 internal -omes that respond to the external environment. To this end, we performed inter-omics analyses

290 between the exposome and gut microbiome, proteome, and metabolome, respectively. By discovering high-

291 degree components in each analysis, we identified the critical components in the exposome-internal omes

292 interactions. For instance, we found six highest-degree gut bacteria that may be important in the responses

293 to the personal exposome. The high-degree biological components in both exposome-proteome and

294 exposome-metabolome analyses were mainly fungi, yet few had known human health effects. However,

295 we identified major high-degree annotated chemicals that are known human stressors; for instance, the

296 herbicide dinoseb exposure causes various developmental toxicities and loss of thyroid and body weight

297 [38], and brominated flame retardants like tetrabromobisphenol A diallyl ether are known neurotoxicants

298 [39]. In addition to the endogenous metabolites, we were able to annotate 60 toxins and carcinogens in the

299 individual's blood based on the exposome related databases (Figure 1c). Unlike the annotated xenobiotics

300 in the exposome samples, most of the chemical stressors annotated in the blood were food and animal

301 toxins. Furthermore, only 11 chemical stressors were annotated in both the external and blood exposome.

302 This is likely to be partly due to the limited power of the current databases, since most of the databases only

303 contain the information of parent chemicals but not their biotransformation products. Additionally,

304 persistent hydrophobic substances tend to accumulate in adipose tissues, but not in blood (which we

305 profiled), while non-persistent hydrophilic chemicals are efficiently excreted out of the human body 
$306[13,16]$, limiting their detections. Finally, the bioavailability of chemicals in different external matrices also

307 limits the exposure, dose-response, and concentration of bioavailable fraction [35,40,41].

Importantly, our results indicate that the immune system, kidneys, and liver may play essential roles in

310 response to the exposome, which are all known to regulate and respond to foreign substances $[17,42,43]$.

311 In the exposome-proteome analysis, we found 14 out of 17 high-degree proteins were involved in immune

312 responses (e.g., complement component 3, interleukin-1 receptor accessory protein, and immunoglobulin

313 heavy chain proteins) [43], and immune-related pathways (e.g., acute inflammatory response, humoral

314 immune response, and complement and coagulation cascades) and were among the highest-degree signaling

315 pathways. In the exposome-metabolome analysis, we identified 19 highest-degree metabolites related to

316 protein metabolism, inflammation, kidney and liver functions (e.g., L-arginine, nutriacholic acid, epsilon-

317 (gamma-Glutamyl)-lysine, and uracil), indicating that these metabolic pathways were involved in responses

318 to the exposome. Moreover, certain high-degree metabolic pathways are both protein and immune-related

319 pathways, such as alanine aspartate and glutamate metabolism, protein digestion and absorption, and beta-

320 alanine metabolism. Specifically, particular protein metabolism pathways (e.g., amino acids synthesis and

321 protein breakdown) were highly sensitive to oxidative stresses caused by the exposome components [44],

322 inflammation is often the first immunological response to foreign substances, and kidneys and liver are the

323 main detoxification organs [44], with liver and bile acids serving essential roles in responding to the foreign

324 substances [45].

326 To further investigate the health effects of the exposome, we performed Spearman correlation analysis with

327 cytokines and blood test results. Proinflammatory cytokines were the most significantly correlated with the

328 external exposome components (e.g., IL-23, MCP-1, and IL-2), and they have been previously shown to be

329 elevated after exposure to external stressors [46]. Our blood test results provided further evidence as

330 fluctuations of creatinine and urea nitrogen, biomarkers of kidney and liver functions, respectively, were

331 correlated with specific exposome components. As a result, exposome-proteome analysis cross-validated 
332 with exposome-cytokine analysis, indicating that the proinflammatory processes play essential roles in

333 responding to the exposome, and the exposome-metabolome analysis cross-validated with exposome-blood

334 tests analysis, showing that liver and kidneys play significant roles in responding to the exposome.

335 Furthermore, the exposome-microbiome analysis showed that the highest-degree gut bacteria are related to

336 the proinflammatory processes and liver metabolism. Therefore, these physiological processes and organs

337 may be ideal candidates for testing the combined effects of multiple stressors in future studies.

339 On the exposome side, high-degree exposome components that overlapped in more than one inter-omics

340 analysis are significant health concerns. Specifically, Isaria, Sporothrix, and Tarenaya were among the

341 highest-degree microbes correlated with all internal -omes. Members of these genera were found to be

342 involved in complex physiological mechanisms and may exhibit adverse health effects. For example,

343 species of Isaria were found to induce cell death [47], species of Sporothrix triggered skin and lung

344 inflammatory reactions [48], and the pollen of Tarenaya members are allergens and generate

345 immunoglobulin-E mediated allergic reactions [49]. Also, certain types of agrochemicals, PPCPs, and

346 flame retardants that we detected are high-degree chemical stressors known to cause epigenetic alterations,

347 endocrine disruption, impaired nervous system function, oxidative stress, and inflammation [44]. Therefore,

348 those substances would be ideal candidates for investigating the underlying mechanisms of their combined

349 health effects in future studies. Such information may be especially important for understanding

350 environmental triggers for inflammatory diseases such as inflammatory bowel diseases, autoimmune

351 diseases, and skin inflammation.

353 In conclusion, we found both time and location impacted the personal exposome, especially the biological

354 components and environmental factors (Figure S2f), and the biological and chemical exposome was highly

355 dynamic. These results emphasize the significance of individual precision environmental health over

356 traditional environmental epidemiology studies. Undoubtedly, due to the limitation of the single participant

357 and annotation power in this study, future research should monitor the precision environmental health of 
358 more individuals and increase the annotation confidence of the chemical and biological components in the

359 exposome. In addition, we focused on the airborne exposome and did not measure other exposures, such as

360 dermal and ingestion exposures, inorganic chemical components [50-53], psychosocial stressors, as well

361 as personal lifestyle, which may affect the clinical measurements as well (Figure 6a). Nonetheless, this

362 study demonstrates the power of using a holistic approach of monitoring the exposome on personal

363 environmental health using inter-omics analyses, and serves as a useful model to scale to the other

364 individuals and locations. Our study also identified high-degree components as essential components

365 among the exposome-internal omes interactions and provided abundant testable hypotheses to further

366 investigate their underlying mechanisms of impacting individual health. 

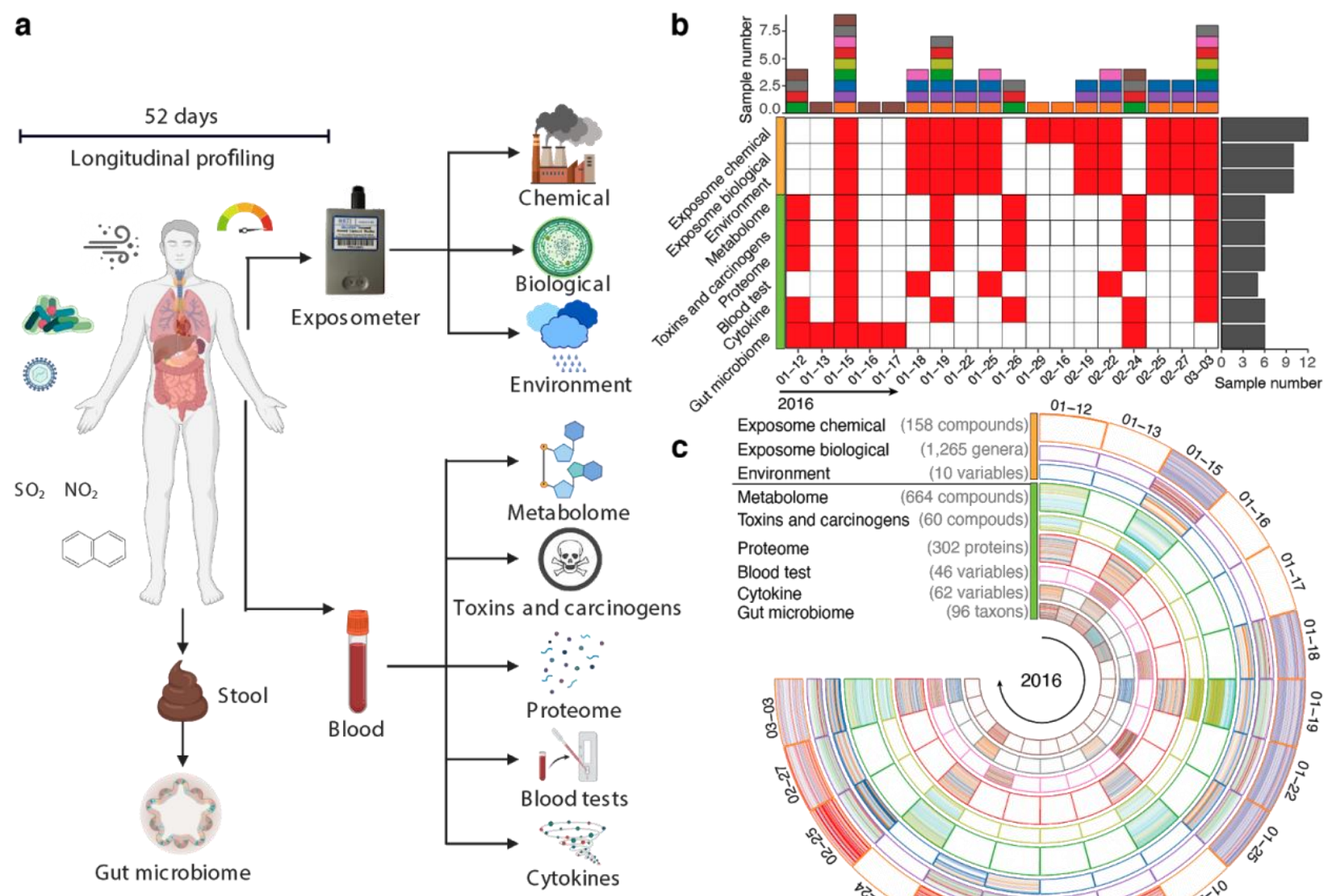

C Exposome chemical (158 compounds) $\left.\right|^{01-12}{ }^{01-13}$

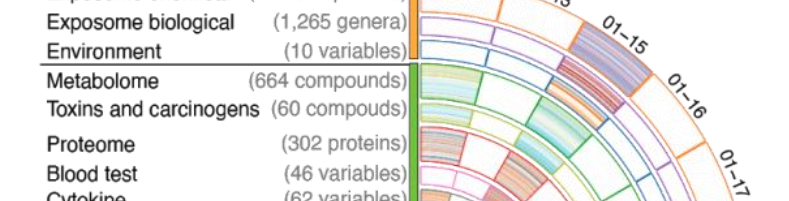

Cytokine

Gut microbiome

(62 variables)

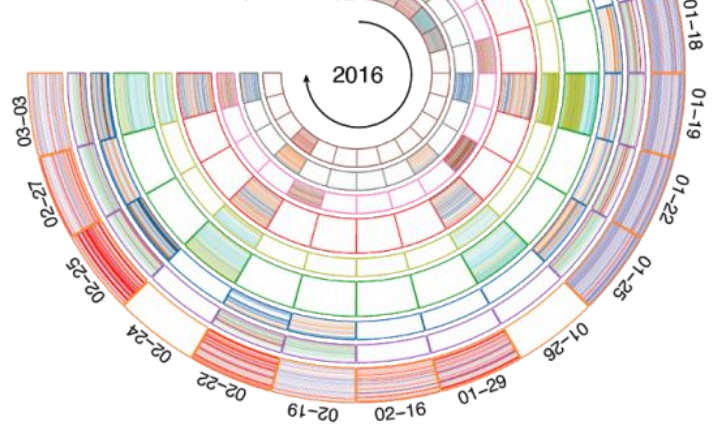

368 Figure 1. Overview of longitudinal sample collections for personal exposome and multi-omics profiling.

369 (a) Personal exposome characterized by the exposometer includes environmental factors, biological

370 components, and chemical stressors. Internal multi-omes include gut microbiome, metabolome, proteome,

371 toxins and carcinogens, cytokines, and blood tests. (b) The amount and collection time of each type of

372 multi-omics and exposome samples. (c) Sample distribution and constitution of the exposome and internal

373 multi-omics for monitoring precision environmental health. 

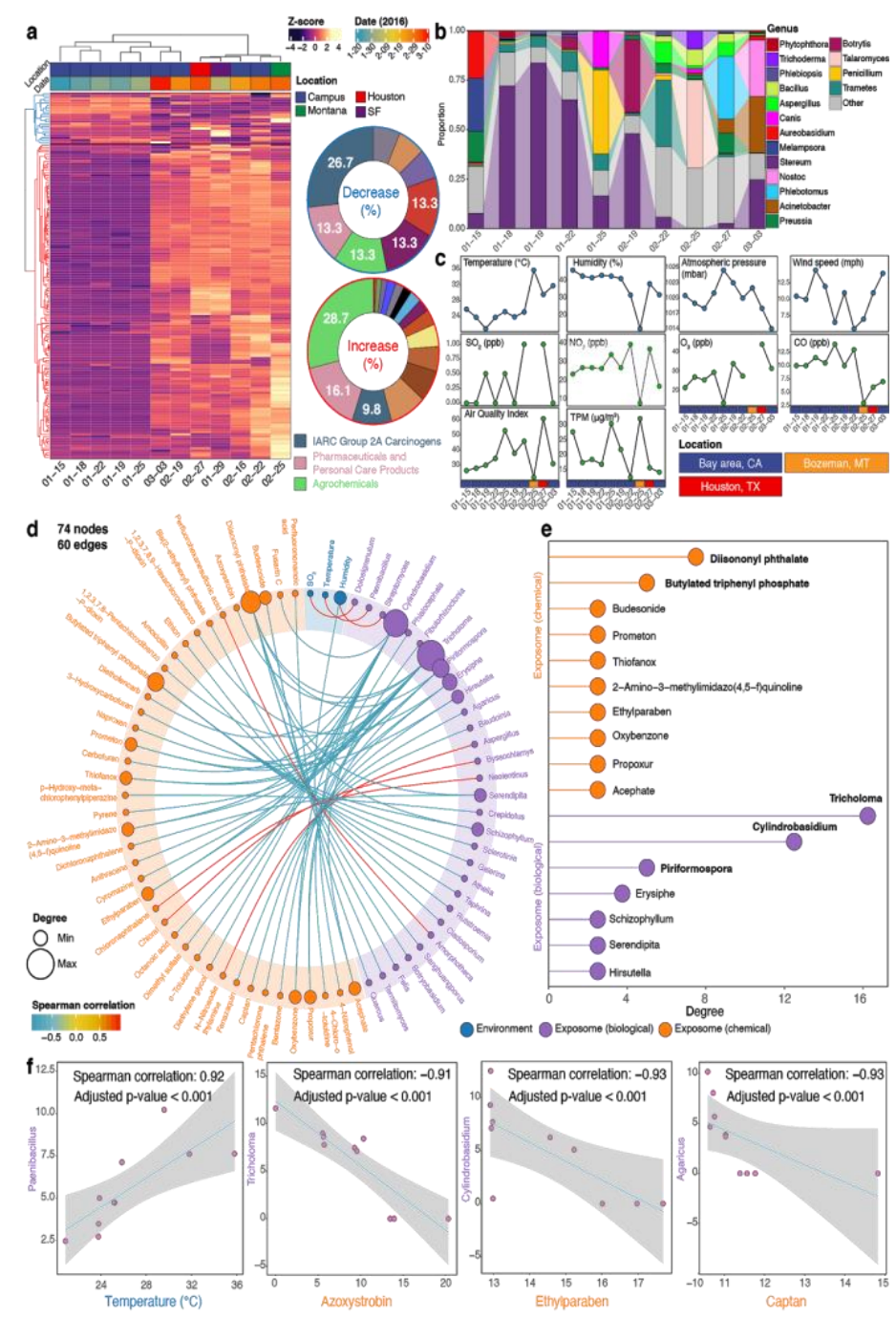

375 Figure 2. The dynamic and diverse personal exposome cloud. (a) Heat map of the annotated chemical 376 stressors in the exposome ordered by concentrations, with the sector diagrams indicating the increased and 377 decreased chemical groups. The abrupt concentration increase after the January 25 sample indicates the 378 approach can monitor dramatic changes of the chemical exposome. (b) Heat map of the top abundant genera 379 annotated in the exposome during each collection period. (c) Environmental factors collected by either the 380 personal exposometer (temperature, humidity, total particulate matter) or local monitoring stations. TPM: 381 total particulate matter. (d) Spearman correlation analyses within the personal exposome $[|r|>0.9$; False 382 Discovery Rate (FDR) adjusted p-value (q-value) < 0.05]. (e) Chemical and biological components that 383 have the most significant correlations with the other substances in the exposome. (f) Representative 384 Spearman correlation analyses between fungi and temperature/antifungal chemicals. 

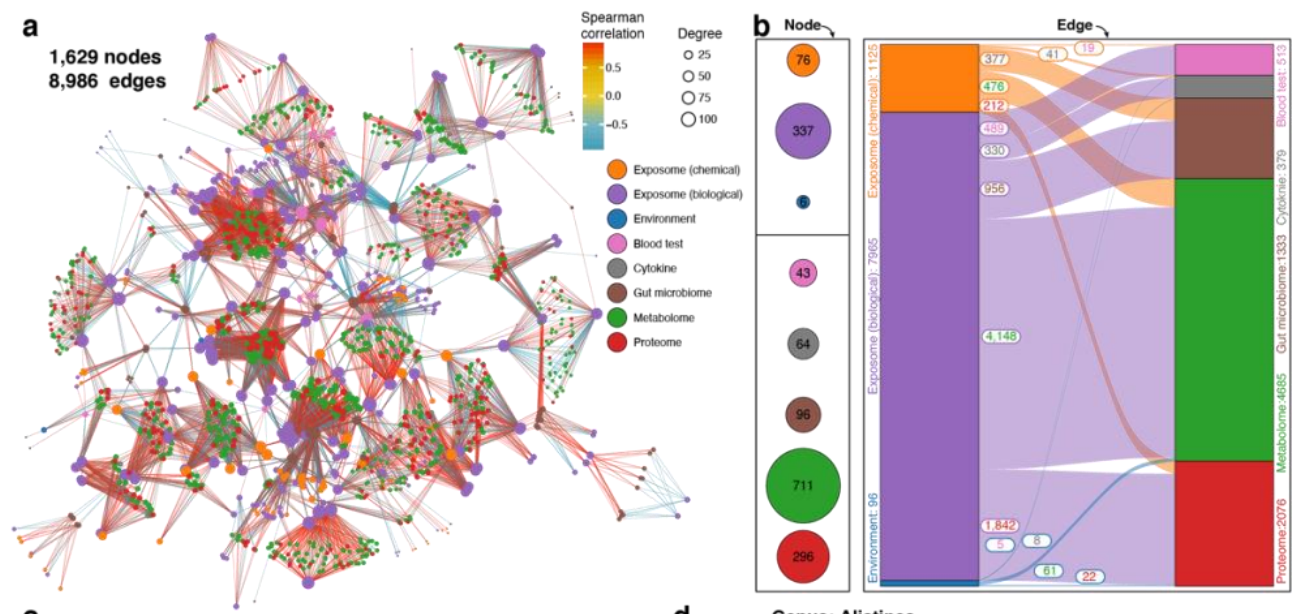

$$
\text { c }
$$

d

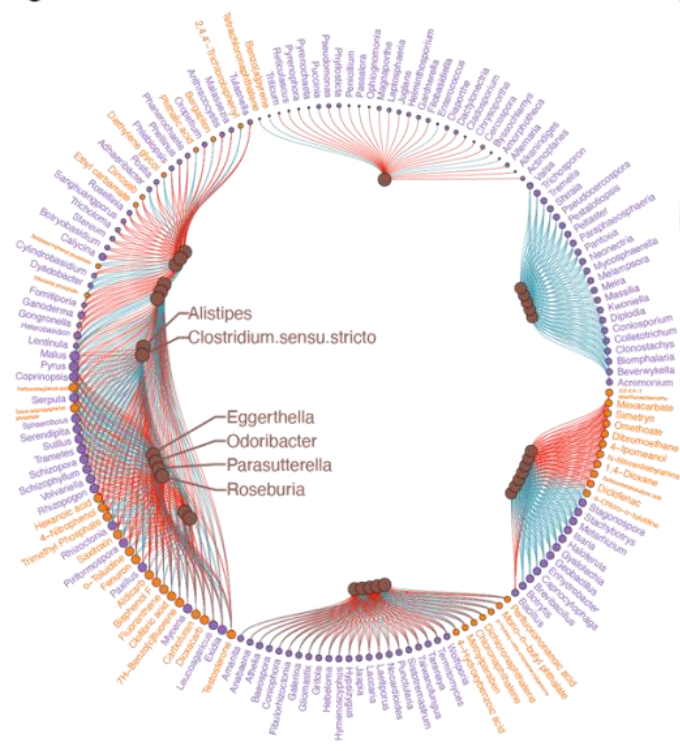

Genus: Alistipes

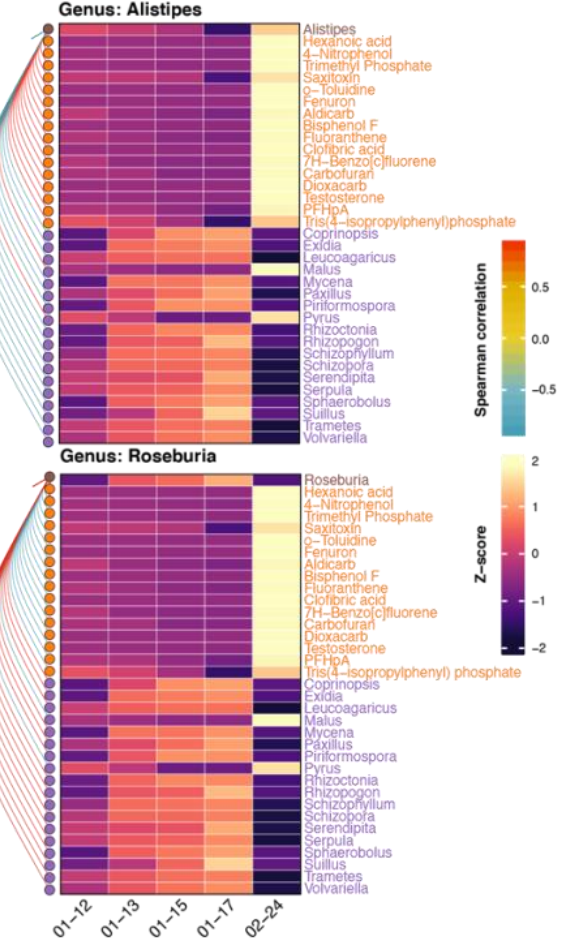

386 Figure 3. Precision environmental health network revealed by inter-omics analyses between the exposome

387 and internal multi-omics. (a) Spearman correlation network of all longitudinally profiled exposome and

388 internal -omes. (b) Significant Spearman correlations between the exposome and the internal multi-omics

$389(|\mathrm{r}|>0.9 ; \mathrm{q}$-value < 0.05). (c) Spearman correlation analysis between the individual's exposome and the gut

390 microbiome. Only gut bacteria with degrees $>20$ are shown, and the highest-degree bacteria are named.

391 The complete network is provided in Figure S4c. (d) Heat map of the highest-degree personal gut bacteria

392 and significant correlations with the exposome components $(|r|>0.9$; q-value $<0.05)$. Additional high-

393 degree gut bacteria are provided in Figure S4d. 

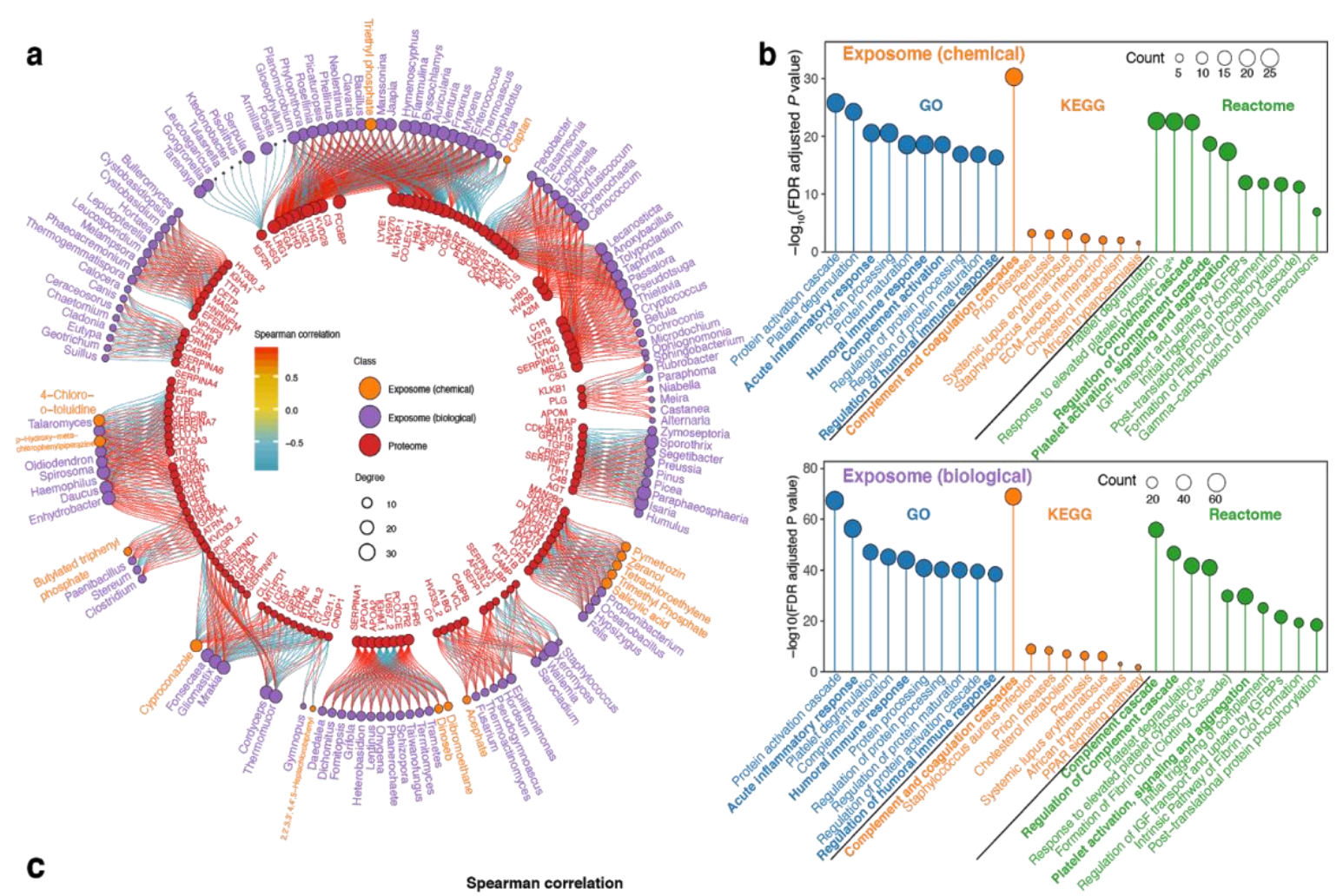

C
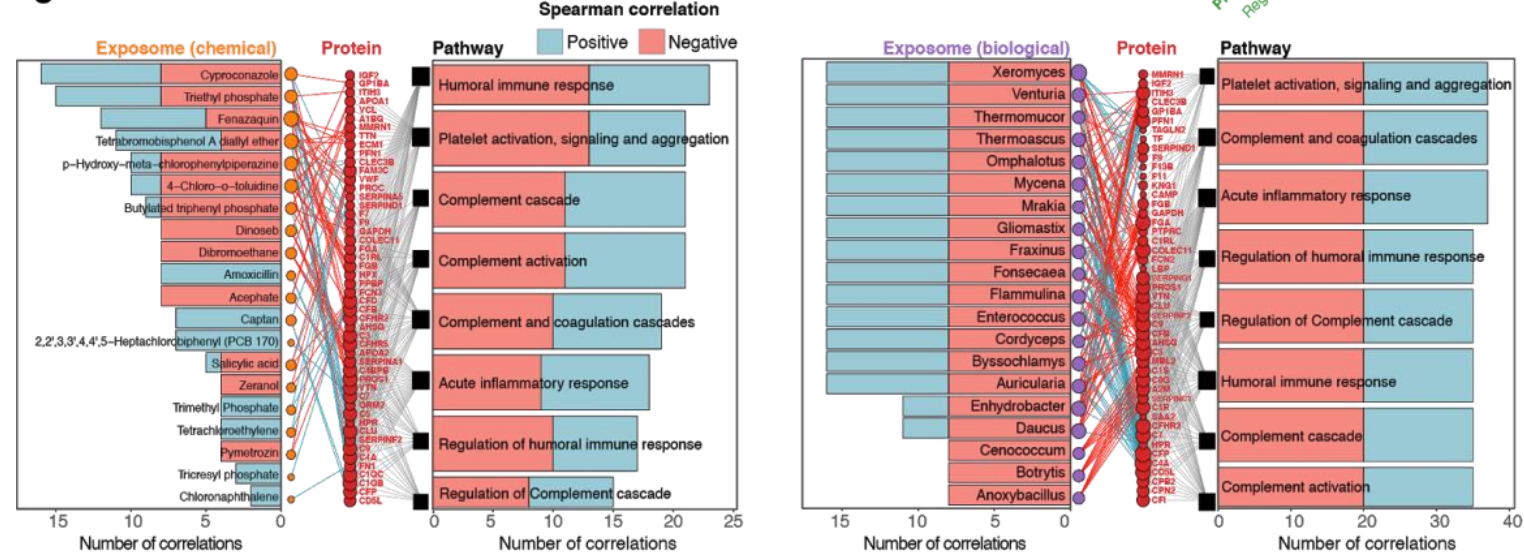

Figure 4. Exposome-proteome interactions: proteins and signaling pathways that were significantly correlated with the exposome. (a) Spearman correlation analysis between the exposome and proteome $(|\mathrm{r}|>$ 0.9 ; q-value $<0.05)$. Only the proteins with degree $>5$ are shown. The complete network is provided in Figure S5. (b) Signaling pathways that significantly correlated with the exposome revealed by pathway analysis using KEGG, GO, and Reactome databases. Immune-related pathways are shown in bold. (c) Spearman correlation networks between chemicals, top twenty biological exposome components, immunerelated proteins and signaling pathways $(|\mathrm{r}|>0.9$; q-value $<0.05)$, with positive correlations shown in blue and negative correlations shown in red. A detailed network for each pathway was provided in Figure S6. 

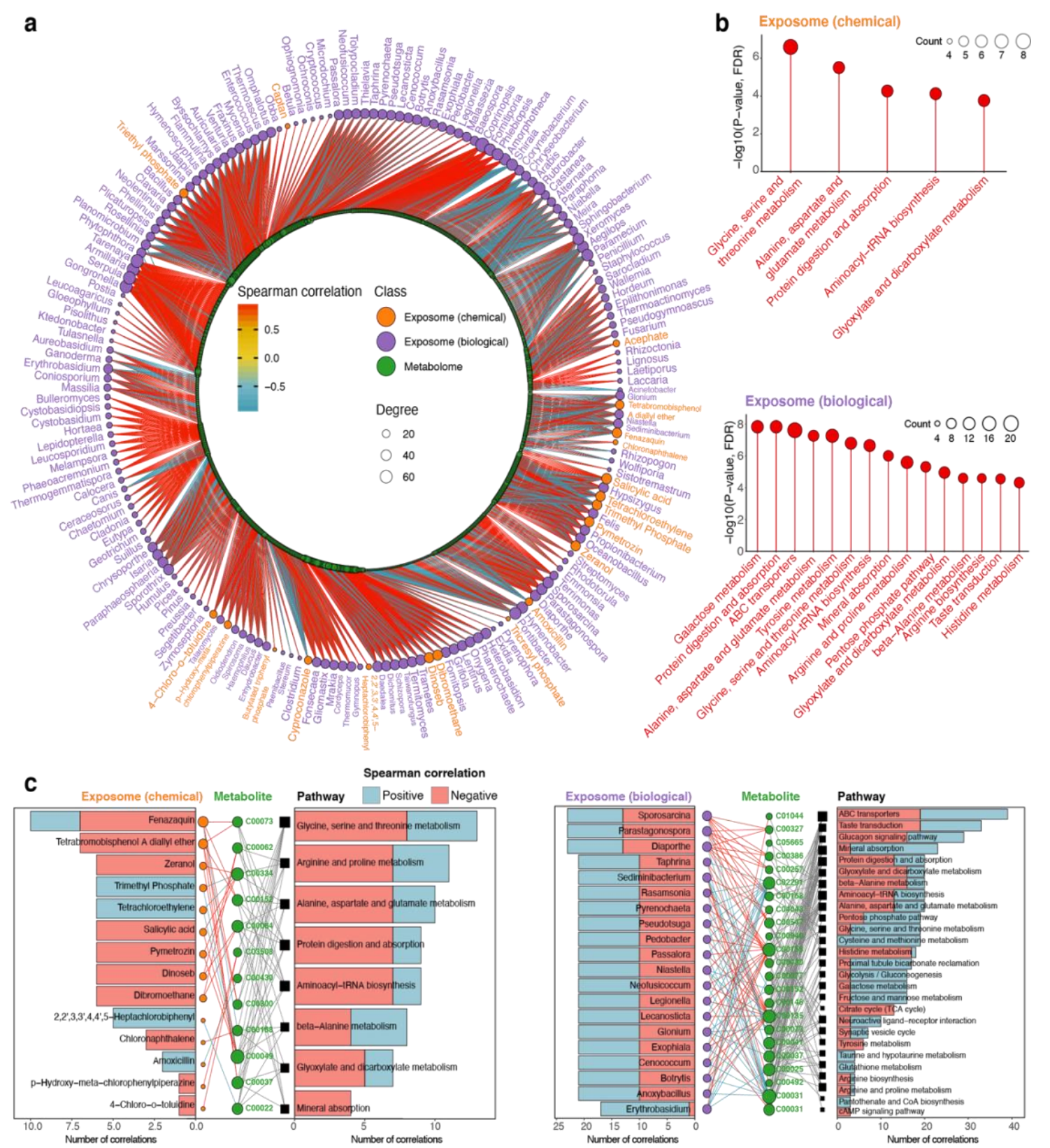

404 Figure 5. Exposome-metabolome interactions: metabolites and metabolic pathways that were significantly

405 correlated with the exposome. (a) Spearman correlation analysis between the exposome and metabolome.

406 (b) Significantly correlated metabolic pathways revealed by pathway analysis using KEGG database $(|\mathrm{r}|>$

407 0.9; q-value <0.05). (c) Significant correlations between chemicals and top twenty biological exposome

408 components and metabolites (represented by KEGG compound entry) and metabolic pathways revealed by

409 Spearman correlation networks $(|\mathrm{r}|>0.9$; q-value $<0.05)$, with positive correlations shown in blue and

410 negative correlations shown in red. The complete network was provided in Figure S8. 

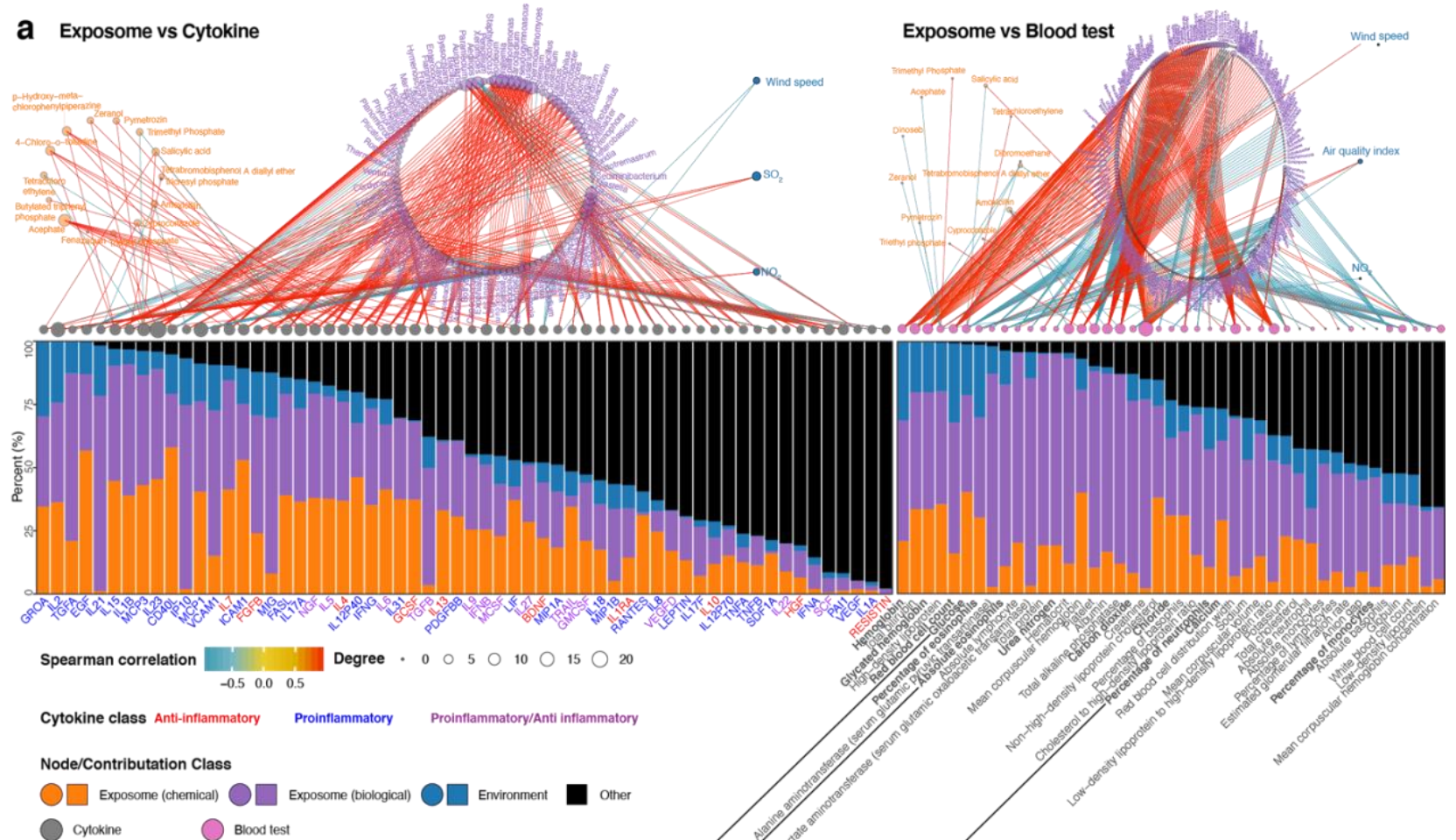

b

\section{Glucose}

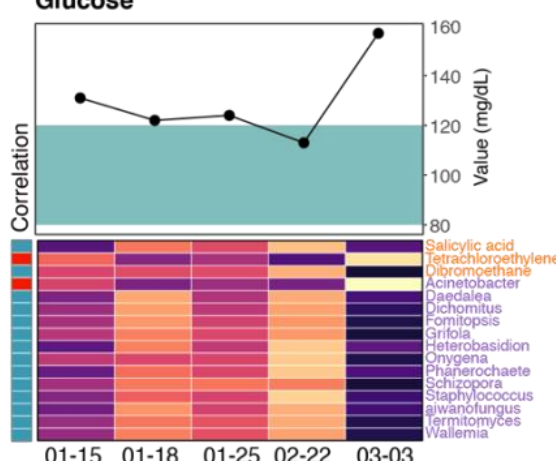

$\begin{array}{lllll}01-15 & 01-18 & 01-25 & 02-22 & 03-03\end{array}$ $\stackrel{2016}{\longrightarrow}$

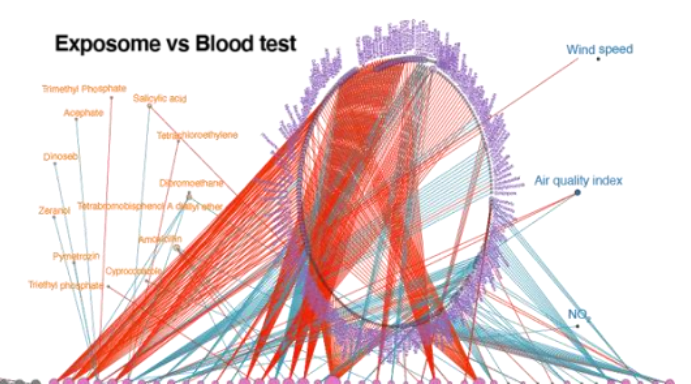




\section{Methods}

\section{Personal exposome and internal multi-omics samples collection}

\section{Exposome samples collection}

419 The participant in the study is enrolled under Stanford University's IRB protocols IRB-23602 and IRB-

420 34907. Modified RTI MicroPEM V3.2 personal exposure monitor (RTI International, Research Triangle

421 Park, North Carolina, USA), termed exposometer, was used to collect chemical and biological components

422 exposed by individuals from January 2016 to March 2016. Also, temperature, humidity, and total particulate

423 matter were simultaneously collected by the exposometer in a real-time manner. The original sequential

424 oiled frit impactor was removed to maximize the collection of biological components. A $0.8 \mathrm{~mm}$ pore-size

425 polyethersulfone with a diameter of $25 \mathrm{~mm}$ filter (Sterlitech, Kent, Washington, USA) was placed in filter

426 cassettes to collect particulates for DNA and RNA extraction. An in-house designed, 3D printed cartridge

427 was placed at the end of the airflow, which contained $200 \mathrm{mg}$ of zeolite adsorbent beads (Sigma 2-0304) to

428 collect chemicals. Before deployment to the participant, the MicroPEM was calibrated to a flow rate of 0.5

$429 \mathrm{~L} / \mathrm{min}( \pm 5 \%)$ using a mass flow meter (TSI 4140, Shoreview, Minnesota, USA). During the study, the

430 participant was instructed to place the exposometer on his arm or within a radius of 2 meters. Samples were

431 collected after 1 to 3 days of use and stored at $-80{ }^{\circ} \mathrm{C}$ until analysis. To minimize the potential

432 contamination, filters and related components were handled in sterile biological safety cabinets and cleaned

433 by ethanol before use. Clean polyethersulfone filter and zeolite adsorbent beads were included before

434 extraction as background controls. MicroPEM log files were downloaded using Docking Station software

435 (RTI International, Research Triangle Park, North Carolina, USA). The participant used the MOVES App

436 to track geographic locations through GPS coordinates and daily activities [10]. General environmental data

437 were collected from the exposometer or National Oceanic and Atmospheric Administration's National

438 Climatic Data Center or National Centers for Environmental Information. 

(4.6 mm x $50 \mathrm{~mm}$ ) with a guard column $(4.6 \mathrm{~mm}$ x $10 \mathrm{~mm}$; Shodex, Showa Denko, Tokyo, Japan). The

444 column temperature was maintained at $45{ }^{\circ} \mathrm{C}$ and the sample chamber at $4{ }^{\circ} \mathrm{C}$. The binary mobile phase

445 solvents were: A, $10 \mathrm{mM}$ ammonium acetate in 50:50 (vol/vol) acetonitrile: water; $\mathrm{B}, 10 \mathrm{mM}$ ammonium

446 acetate in 90:10 ( $\mathrm{vol} / \mathrm{vol})$ acetonitrile: water. Both solvents were modified with $10 \mathrm{mM}$ acetic acid ( $\mathrm{pH}$

$4474.75)$ for positive mode acquisition or $10 \mathrm{mM}$ ammonium acetate $(\mathrm{pH} 9.25)$ for negative mode. The flow

448 rate was set as follows: flow rate, $0.1 \mathrm{ml} / \mathrm{min}$; gradient, $0-15 \mathrm{~min}, 99 \% \mathrm{~A}, 15-18 \mathrm{~min}, 99 \%$ to $1 \% \mathrm{~A} ; 18-$

44924 min, $1 \%$ A; 24-25 min, $1 \%$ to $99 \%$ A; 25-30 min, $99 \%$ A. The MS acquisition was set as full scan mode

450 with an ESI probe or APCI probe. The capillary temperature was $275^{\circ} \mathrm{C}$, the sheath gas was 40 units, the

451 positive mode spray voltage was $3.5 \mathrm{kV}$, and $3.1 \mathrm{kV}$ for the negative mode. The capillary voltage was 30

$452 \mathrm{~V}$, the tube lens voltage was $120 \mathrm{~V}$, and the skimmer voltage was $20 \mathrm{~V}$. The mass spectrum scan used

453100,000 mass resolution, high dynamic range for AGC target, maximum injection time of $500 \mathrm{~ms}$, and a

454 scan range of $70-1,000 \mathrm{~m} / \mathrm{z}$. The details of quality assurance and quality control were described in the 455 previous study [54].

457 Post-acquisition analysis of the chemical exposome

458 Analysis of chemical exposome was performed as previously described [10,54]. In brief, feature detection 459 was performed with XCMS. For a conservative assessment of the number of unique chemical features, a 460 customized Python script was used to remove potential isoforms, isotopes, and adducts from the 3,299 461 features enriched at least 10-fold as compared with the blank control. The annotation was based on various 462 exposome related databases that are publicly available as well as in-house databases [55-57]. The 463 annotation confidence levels of all the chemicals in the exposome were at least level 5, with at least one 464 chemical at level 1 or 2 in each chemical class [54,58]. 
DNA and RNA sequencing and analysis were performed as previously described [10,54]. In brief, DNA and RNA were extracted from filters and linearly amplified for sequencing. Libraries were sequenced by Illumina HighSeq 4000 platform ( 2 x 151bp) that yields an average of $\sim 50 \mathrm{M}$ unique reads per sample. Sequenced reads were deduplicated, and adapters were trimmed using Trim Galore! (version 0.4.4). Human

471 related reads were identified using BWA mapped to the hg19 human genome and removed. Following

472 dehumanization, non-human reads were used for de-novo assembly using Megahit (1.1.1), and contigs were

473 queried against our in-house database with BLASTN (2.3.9+) wrapper. The extensive in-house reference

474 genome database included more than 40,000 species covering all domains of life [10]. Taxonomy

475 classification and abundance were determined using a customized Lowest Common Ancestor (LCA) 476 algorithm.

\section{Blood sample collection}

479 At the designated time point, blood was drawn from the overnight fasted participant in the Clinical and

480 Translational Research Unit at Stanford University. Aliquots of blood were condensed at room temperature

481 to coagulate, and clots were subsequently pelleted. The serum supernatant was then immediately frozen at

$482-80{ }^{\circ} \mathrm{C}$. The blood in the EDTA tubes was immediately layered onto the Ficoll medium and spun with

483 gradient centrifugation. Then the top layers were removed, and plasma was aliquoted and immediately

484 frozen at $-80{ }^{\circ} \mathrm{C}$. Subsequently, blood mononuclear cells (PBMCs) were collected and counted using a cell

485 counter. Aliquots of PBMCs were further pelleted and frozen with DMSO/FBS. For the later multi-omics

486 analyses, PBMCs were thawed on ice and then lysed to protein fraction using Allprep Spin Columns

487 (Qiagen) according to the manufacturer's instructions with the QIAshredder lysis option. Upon receipt of 488 samples, blood samples were then stored at $-80^{\circ} \mathrm{C}$ for clinical tests. The details of the blood and cytokines 489 tests can be found in Supplementary Data 1 [15]. 
492 Stool samples were collected according to the Human Microbiome Project-Core Microbiome Sampling

493 Protocol A (https://www.hmpdacc.org/). Following the Human Microbiome Project-Core Microbiome

494 Sampling Protocol A (HMP Protocol \#07-001, v12.0), DNA extraction was performed. We used the

495 MOBIO PowerSoil DNA extraction kit and proteinase K to isolate DNA in a clean fume hood. Samples

496 were then treated with lysozyme and staphylococcal hemolysin. For 16S (bacterial) rRNA gene

497 amplification, primers 27F and 534R (27F: 5'-AGAGTTTGATCCTGGCTCAG-3' and 534R: 5'-

498 ATTACCGCGGCTGCTGG-3') were used to amplify the 16S hyper-variable regions V1-V3. Unique

499 barcode amplicons were used, and samples were sequenced on the Illumina MiSeq platform (V3; $2 \times 300$

500 bp). Illumina software handled the initial processing of all raw sequencing data. Reads were further

501 processed by removing low-quality (average quality <35) and ambiguous base (Ns) sequences. UChime

502 was used to remove chimeric amplicons, cluster the amplicon sequences, and select the operational

503 taxonomic unit by Usearch based on the GreenGenes database (version in May 2013). The final biological

504 classification assignment was performed using the RDP-classifier in QIIME with custom scripts [14].

\section{Untargeted proteomics by LC-HRMS}

507 Preparation and analysis of plasma samples were performed as previously described [14]. In short, tryptic 508 peptides from plasma samples were separated on the NanoLC 425 system (SCIEX). $0.5 \times 10 \mathrm{~mm}$ ChromXP 509 (SCIEX) was used for trap-elution settings, and the flow rate was set to $5 \mu \mathrm{l} / \mathrm{min}$. The LC gradient was a 510 43-minute gradient with mobile phase A: $0.1 \%$ formic acid in $100 \%$ water and mobile phase B: $0.1 \%$ formic 511 acid in $100 \%$ acetonitrile. During the gradient, mobile phase B was 4-32\%. Then $8 \mu \mathrm{g}$ of undepleted plasma 512 were loaded on LC. SWATH acquisition was performed on a TripleTOF 6600 system equipped with a 513 DuoSpray source and a $25 \mu \mathrm{m}$ ID electrode (SCIEX). The variable Q1 window SWATH acquisition mode

514 (100 windows) was constructed in the high-sensitivity $\mathrm{MS}^{2}$ mode. PyProphet [59] was used to score the 515 peak groups in each run statistically, and TRIC [60] was used to align all runs. Finally, a matrix with a 516 peptide level of $1 \%$ FDR and a protein level of 10\% FDR was generated for subsequent analysis. The 
protein abundances were the sum of the first three most abundant peptides. Perseus (v 1.4.2.40) was applied to subtract the main components showing the main batch deviation to reduce batch effect [14].

\section{Cytokine profiling}

521 The levels of circulating cytokines in the blood were measured by the 62-plex Luminex antibody522 conjugated magnetic bead capture assay (Affymetrix), which has been extensively characterized and 523 benchmarked by the Stanford Human Immunological Monitoring Center. The human 62-plex 524 (eBiosciences/Affymetrix) was utilized with the modifications described below. Briefly, beads were added 525 to a 96-well plate and washed using Biotek ELx405. Samples were added to the plate containing mixed 526 antibody-linked beads, incubated at room temperature for 1 hour, and then overnight at $4{ }^{\circ} \mathrm{C}$ with shaking 527 (500-600 r.p.m, orbital shaker). After overnight incubation, the plate was washed, and then the biotinylated antibody was added. The plate was incubated at room temperature with shaking for 75 minutes. The plate was washed, and streptavidin-PE was added for detection. After incubating for 30 minutes at room temperature, the plate was washed once, and then the reading buffer was added to the wells. The plate was read by a Luminex 200 instrument, and the lower limit of each cytokine per sample was set to 50 beads. Radix Biosolutions custom assay control beads were added to all wells. The batch effect was corrected using replicates and controls shared between batches [14].

\section{Untargeted metabolomics by LC-HRMS}

536 All blood samples were prepared and analyzed for metabolomics as previously described [61]. In short,

537 plasma samples were extracted with acetone: acetonitrile: methanol (1:1:1 vol/vol/vol), evaporated to 538 dryness under nitrogen and reconstituted in methanol: water (1:1 vol/vol) for LC-HRMS analysis. HILIC 539 and RPLC separations were used to analyze the extractants four times in positive and negative modes, 540 respectively. HILIC metabolomics data was obtained on a Thermo Q Exactive plus, and RPLC 541 metabolomics data was obtained on a Thermo Q Exactive. Both instruments were equipped with HESI-II 542 probes and operated in the full MS scan mode. We only used the combined quality control samples from 
543 the study to obtain $\mathrm{MS}^{2}$ data. We used a ZIC-HILIC column $(2.1 \times 100 \mathrm{~mm}, 3.5 \mu \mathrm{m}, 200 \AA ̊$; Merck

544 Millipore) and mobile phases composed of $10 \mathrm{mM}$ ammonium acetate in acetonitrile: water (50:50 vol/vol)

545 (A) and $10 \mathrm{mM}$ ammonium acetate in acetonitrile/water (95:5 vol/vol) (B), and a Zorbax SB-Aq column

$546(2.1 \times 50 \mathrm{~mm}, 1.7 \mu \mathrm{m}, 100 \AA$; Agilent Technologies $)$ and mobile phases composed of $0.06 \%$ acetic acid in

547 water (A) and $0.06 \%$ acetic acid in methanol (B) to perform HILIC and RPLC analyses, respectively. All

548 raw metabolomics data were processed using Progenesis QI (Nonlinear Dynamics, Waters). We also

549 removed features that did not show sufficient linearity when diluted. Only features presented in more than

$5501 / 3$ samples were retained for further analysis, and the KNN method was used to estimate missing values.

551 To normalize the data, locally assessed scatter plot smoothness analysis was applied [62]. Metabolic

552 signatures were identified by matching retention time and fragmentation spectra to corresponding standards

553 or comparing fragmentation patterns to public repositories, as previously reported [14]. Toxin and

554 carcinogens were annotated out of the metabolome features if the feature cannot be annotated as a human

555 metabolite. The annotations of toxins and carcinogens were based on various blood exposome related

556 databases that are publicly available as well as in-house databases [55-57]. The confidence levels of blood

557 chemical annotation were at least level 5, with the majority at least level 3 [58].

\section{General statistical analysis and data visualization}

560 All statistical analysis and data visualization were performed using R (v3.6.0, https://www.r-project.org/)

561 and RStudio (v 1.2.5019). Most of the R packages and their dependencies used in this study were deployed

562 in CRAN (https://cran.r-project.org/) or Bioconductor (https://bioconductor.org/), and some of them are

563 deployed on Github. Session information for this study is provided in Supplementary Note 1. All scripts

564 to reproduce analysis and data visualization for this study is available on Github

565 (https://github.com/jaspershen/precision_exposome/tree/main/R/20200511). All data from the exposome

566 and internal -omes data were $\log 2$ transformed before analysis. According to the participant's food log,

567 fiber intake was adjusted for all internal -omes data to reduce fiber intake biases (Supplementary Note 2). 


\section{Exposome and internal multi-omics correlation networks}

570 Spearman correlation was used to build the correlations in the intra/inter-omics analyses. In general, for

571 each two -omes pair, the correlation matrix was calculated as below: for each variable in one -omes,

572 Spearman correlations and FDR adjusted p-values were generated with all features in the other -omes. Only

573 correlations between each pair variable with absolute correlation $>0.9$ and FDR adjusted $p$-value $<0.05$

574 were kept to construct the final correlation networks.

\section{Community analysis}

577 Community analysis was performed based on edge betweenness embedded in $\mathrm{R}$ package igraph 578 (https://igraph.org/). Briefly, this is an iterative process, the edges with the highest edge betweenness score 579 were removed in each iteration, and the process was repeated until only individual nodes remain. At each 580 iteration, modularity was calculated, and communities were analyzed at the iteration that maximized this 581 quantity. A visualization of iteration community versus modularity is shown in Figure S6 a, b. To ensure the robustness and reliability of our findings, only communities (or clusters) with at least 3 nodes were kept

583 for subsequent analysis. All the networks were visualized using R package igraph, ggraph and tidygraph.

\section{GO, KEGG and Reactome pathway enrichment for proteome}

586 The R package ClusterProfiler $(\mathrm{v}$ 3.18 .0

$587 \mathrm{https} / / /$ bioconductor.org/packages/release/bioc/html/clusterProfiler.html) was used for GO, KEGG and 588 Reactome pathway enrichment for proteomics. In general, UNIPROT and ENTREZID were obtained for 589 proteins that connect with the exposome in correlation networks. Then the GO, KEGG and Reactome

590 pathway databases were used for pathway enrichment (hypergeometric distribution test, $p$-values are 591 adjusted by the FDR method, and the cutoff was set as 0.05$)$. Only pathways with hitting protein number > 5923 were retained for subsequent analysis.

594 Metabolic feature based dysregulated module detection 

community analysis were utilized to detect dysregulated modules based on metabolic features connecting the exposome, respectively [63]. In general, the metabolic network (MN) was downloaded from KEGG, which contains 1,377 nodes (metabolites) and 1561 edges (reactions). The brief workflow is described 599 below:

600 1. All the metabolic features connecting the exposome $\left(\mathrm{L}_{\mathrm{sig}}\right)$ were matched with the KEGG metabolite database based on different adducts (Supplementary Table 1). Then all matched metabolites (significant metabolites) are mapped in the metabolic network to get the subnetwork (SN). Nonsignificant metabolites (hidden metabolites) that can connect significant metabolites within 3 reactions were also included in the subnetwork. Then the modules $(\mathrm{M})$ were detected in the subnetwork via random walks [65]. Only modules with at least 3 nodes were kept. These modules were named significant modules $\left(\mathrm{M}_{\text {sig }}\right)$ from real biological-related metabolic features.

2. For each module, the activity score (S) was calculated to measure both the modularity and enrichment of input metabolites (I). Activity score (S) of the module (M) was calculated as follows: For a module M: metabolite number in module M. Q is the adjusted Newman-Girvan modularity calculated as below: $\mathrm{Q}=\sqrt{\frac{N_{I}}{N_{M}}} *\left(\frac{E_{M}}{m}-\sum_{i, j} \quad \frac{k_{i}}{2 m} * \frac{k_{j}}{2 m}\right), i, j \in M$ where $\mathrm{k}_{\mathrm{i}}$ is the degree of metabolite $\mathrm{i}$ in module $\mathrm{M}, \mathrm{m}$ is the total number of edges in the metabolic network $M N, E_{M}$ is the total number of edges in module $M$, and $N_{I}$ is the number of input metabolites. The original Newman-Girvan modularity has a bias towards larger modules, and $\sqrt{\frac{N_{I}}{N_{M}}}$ was used to reduce this bias. 
3. Then the NULL metabolic features $\left(\mathrm{L}_{\text {null }}\right.$, the same number with $\mathrm{L}_{\text {sig }}$ ) were selected from all metabolic features (exclude $\mathrm{L}_{\mathrm{sig}}$ ) and then steps 1 and 2 were repeated 100 times to generate a list of NULL modules $\left(\mathrm{M}_{\text {null }}\right)$ and their activity score $\left(\mathrm{S}_{\text {null }}\right)$.

The annotation results from this method were also compared with the annotation results from the

627 showed that annotations from this method have high specificity.

\section{KEGG pathway enrichment analysis for metabolomics data}

The KEGG pathway database was downloaded from KEGG (https://www.genome.jp/kegg/) using the R package KEGGREST. Pathway enrichment analysis was used in the hypergeometric distribution test, and $p$-values were adjusted by the FDR method, and only pathways with FDR adjusted p-value $<0.05$ were

633 kept.

Exposome contributions to cytokine and blood test

636 To calculate the contributions of the exposome on each cytokine and blood test, principal components (PCs)

637 were first extracted for each exposome component, and only PCs with cumulative explained variation >

$63880 \%$ were kept. Then the linear regression model was constructed using each cytokine/blood test as y and

639 corresponding exposome component's PCs as $\mathrm{x} . \mathrm{R}^{2}$ was extracted and used to represent the contributions

640 of the exposome to each cytokine/blood test. To calculate the contribution of the exposome components,

641 partial least squares (PLS) and variable important projection (VIP) were calculated. Finally, $\mathrm{R}^{2} * \frac{V I P_{i}}{\operatorname{sum}(V I P)}(\mathrm{i}$

$642 \in$ chemical, biological, and environment) was used to represent the contributions of the exposome 643 components on cytokine/blood tests. 


\section{Data availability}

646 The biological exposome data generated in this and previous studies were deposited to the National Center

647 of Biotechnology Information under bioproject ID PRJNA421162. Some raw data utilized in this study are

648 presented on the NIH Human Microbiome 2 project site (https://portal.hmpdacc.org). Some other raw and

649 processed data are shown on the Stanford iPOP site (http://med.stanford.edu/ipop.html). The processed data

650 used for reproductive analysis can also be found on Github

651 (https://github.com/jaspershen/precision_exposome) and were provided in Supplementary Data 1.

\section{Code availability}

654 All codes used in this study can be found on Github (https://github.com/jaspershen/precision_exposome).

655 Certain in-house tools for this study can also be found on Github (https://github.com/jaspershen/metID).

\section{Acknowledgements}

658 We thank Xin Wang and Xiyan Li for their contributions to generate the raw data of chemical exposome.

659 This work was supported by Leona M. and Harry B. Helmsley Charitable Trust (grant G-2004-03820).

660 S.M.S.-F.R. was supported by an NIH Career Development Award no. K08ES028825. This work used

661 supercomputing resources provided by the Stanford Genetics Bioinformatics Service Center, supported by

662 NIH S10 Instrumentation Grant S10OD023452.

\section{Author contributions}

665 P.G. and M.S. conceived the study. P.G., X.S., and C.J. analyzed the chemical exposome and

666 environmental factors data. P.G., X.S., X.Z., and C.J. analyzed the biological exposome data. P.G., X.S.,

667 and X.Z. analyzed multi-omics data. M.S. supervised the study and provided funding support. P.G.

668 drafted and revised the manuscript. X.S., X.Z., C.J., S.Z., X.Z., S.M.S.-F.R., and M.S. revised the

669 manuscript. 


\section{References}

671 1. Topol EJ. Individualized medicine from prewomb to tomb. Cell. 2014;157: 241-253.

2. Liu Y, Gao P, Su J, da Silva EB, de Oliveira LM, Townsend T, et al. PAHs in urban soils of two Florida cities: Background concentrations, distribution, and sources. Chemosphere. 2019;214: 220227.

3. Li S, Gao P, Zhang J, Li Y, Peng B, Gao H, et al. Sequential dispersive liquid-liquid microextraction for the determination of aryloxyphenoxy-propionate herbicides in water. J Sep Sci. 2012;35: 33893395 .

4. Li S, Li T, Gao P, Lu R, Zhou W, Gao H. Slow-Injection Ultrasound-Assisted EmulsificationMicroextraction for Determination of Phthalate Esters in Water $\dagger$. Journal of Chromatographic Science. 2014. pp. 1127-1134. doi:10.1093/chromsci/bmt154

5. Guo Y, Laux SJ, Burdier M, Gao P, Ma LQ, Townsend TG. Polycyclic aromatic hydrocarbons in

7. de Oliveira LM, Das S, da Silva EB, Gao P, Gress J, Liu Y, et al. Metal concentrations in traditional

8. Jin Z, Berthiaume JM, Li Q, Henry F, Huang Z, Sadhukhan S, et al. Catabolism of (2E)-4-hydroxy2-nonenal via $\omega$ - and $\omega$-1-oxidation stimulated by ketogenic diet. J Biol Chem. 2014;289: 3232732338 .

9. Vermeulen R, Schymanski EL, Barabási A-L, Miller GW. The exposome and health: Where

10. Jiang C, Wang X, Li X, Inlora J, Wang T, Liu Q, et al. Dynamic Human Environmental Exposome
Revealed by Longitudinal Personal Monitoring. Cell. 2018;175: 277-291.e31. chemistry meets biology. Science. 2020. pp. 392-396. doi:10.1126/science.aay3164

11. Sinharay R, Gong J, Barratt B, Ohman-Strickland P, Ernst S, Kelly FJ, et al. Respiratory and cardiovascular responses to walking down a traffic-polluted road compared with walking in a trafficfree area in participants aged 60 years and older with chronic lung or heart disease and age-matched healthy controls: a randomised, crossover study. The Lancet. 2018. pp. 339-349. doi:10.1016/s01406736(17)32643-0

12. Gauthier PT, Norwood WP, Prepas EE, Pyle GG. Metal-PAH mixtures in the aquatic environment: a review of co-toxic mechanisms leading to more-than-additive outcomes. Aquat Toxicol. 2014;154: 253-269.

13. Gao P. The Exposome in the Era of One Health. Environ Sci Technol. 2021. doi:10.1021/acs.est.0c07033 
15. Schüssler-Fiorenza Rose SM, Contrepois K, Moneghetti KJ, Zhou W, Mishra T, Mataraso S, et al. A longitudinal big data approach for precision health. Nat Med. 2019;25: 792-804.

16. Gao P, da Silva E, Hou L, Denslow ND, Xiang P, Ma LQ. Human exposure to polycyclic aromatic hydrocarbons: Metabolomics perspective. Environ Int. 2018;119: 466-477.

17. Xiang P, He R-W, Liu R-Y, Li K, Gao P, Cui X-Y, et al. Cellular responses of normal (HL-7702) and cancerous (HepG2) hepatic cells to dust extract exposure. Chemosphere. 2018;193: 1189-1197.

18. Xiang P, Jia Y, Wang K, Li M-Y, Qin Y-S, He R-W, et al. Water extract of indoor dust induces tight

19. Kaur N, Seuylemezian A, Patil PP, Patil P, Krishnamurti S, Varelas J, et al. Paenibacillus xerothermodurans sp. nov., an extremely dry heat resistant spore forming bacterium isolated from the soil of Cape Canaveral, Florida. Int J Syst Evol Microbiol. 2018;68: 3190-3196.

20. Rodrigues ET, Lopes I, Pardal MÂ. Occurrence, fate and effects of azoxystrobin in aquatic ecosystems: a review. Environ Int. 2013;53: 18-28.

21. European Food Safety Authority (EFSA), Anastassiadou M, Arena M, Auteri D, Brancato A, Bura $\mathrm{L}$, et al. Peer review of the pesticide risk assessment of the active substance captan. EFSA J. 2020;18: e06230.

22. Parker BJ, Wearsch PA, Veloo ACM, Rodriguez-Palacios A. The Genus Alistipes: Gut Bacteria With Emerging Implications to Inflammation, Cancer, and Mental Health. Frontiers in Immunology. 2020. doi:10.3389/fimmu.2020.00906

23. Lau SKP, Woo PCY, Woo GKS, Fung AMY, Wong MKM, Chan K-M, et al. Eggerthella hongkongensis sp. nov. and eggerthella sinensis sp. nov., two novel Eggerthella species, account for half of the cases of Eggerthella bacteremia. Diagn Microbiol Infect Dis. 2004;49: 255-263.

24. Gomez-Arango LF, Barrett HL, McIntyre HD, Callaway LK, Morrison M, Dekker Nitert M, et al. Increased Systolic and Diastolic Blood Pressure Is Associated With Altered Gut Microbiota Composition and Butyrate Production in Early Pregnancy. Hypertension. 2016;68: 974-981.

25. Ju T, Kong JY, Stothard P, Willing BP. Defining the role of Parasutterella, a previously uncharacterized member of the core gut microbiota. ISME J. 2019;13: 1520-1534.

26. Tamanai-Shacoori Z, Smida I, Bousarghin L, Loreal O, Meuric V, Fong SB, et al. Roseburia spp.: a marker of health? Future Microbiol. 2017;12: 157-170.

27. Safran M, Dalah I, Alexander J, Rosen N, Iny Stein T, Shmoish M, et al. GeneCards Version 3: the human gene integrator. Database. 2010. pp. baq020-baq020. doi:10.1093/database/baq020

28. Walker DI, Valvi D, Rothman N, Lan Q, Miller GW, Jones DP. The metabolome: A key measure for exposome research in epidemiology. Curr Epidemiol Rep. 2019;6: 93-103.

29. Zajc J, Gunde-Cimerman N. The Genus Wallemia-From Contamination of Food to Health Threat. Microorganisms. 2018. p. 46. doi:10.3390/microorganisms6020046

30. Rumore MM, Kim KS. Potential role of salicylates in type 2 diabetes. Ann Pharmacother. 2010;44: $1207-1221$. 
31. Shamri R, Xenakis JJ, Spencer LA. Eosinophils in innate immunity: an evolving story. Cell Tissue Res. 2011;343: 57-83.

32. Rutherford JC. The emerging role of urease as a general microbial virulence factor. PLoS Pathog. 2014;10: e1004062.

33. Li K, Gao P, Xiang P, Zhang X, Cui X, Ma LQ. Molecular mechanisms of PFOA-induced toxicity in animals and humans: Implications for health risks. Environ Int. 2017;99: 43-54.

34. Gao P, Xu M, Liu Y, da Silva EB, Xiang P, Ma LQ. Emerging and legacy PAHs in urban soils of four small cities: Concentrations, distribution, and sources. Sci Total Environ. 2019;685: 463-470.

35. Gao P, da Silva EB, Townsend T, Liu X, Ma LQ. Emerging PAHs in urban soils: Concentrations, bioaccessibility, and spatial distribution. Sci Total Environ. 2019;670: 800-805.

36. Xiang P, Liu R-Y, Li C, Gao P, Cui X-Y, Ma LQ. Effects of organophosphorus flame retardant TDCPP on normal human corneal epithelial cells: Implications for human health. Environ Pollut. 2017;230: 22-30.

37. Guo H, Chou W-C, Lai Y, Liang K, Tam JW, Brickey WJ, et al. Multi-omics analyses of radiation survivors identify radioprotective microbes and metabolites. Science. 2020;370. doi:10.1126/science.aay9097

38. Matsumoto M, Poncipe C, Ema M. Review of developmental toxicity of nitrophenolic herbicide dinoseb, 2-sec-butyl-4,6-dinitrophenol. Reprod Toxicol. 2008;25: 327-334.

39. Eriksson P, Jakobsson E, Fredriksson A. Brominated flame retardants: a novel class of developmental neurotoxicants in our environment? Environ Health Perspect. 2001;109: 903-908.

40. Zhang S, Li C, Li Y, Zhang R, Gao P, Cui X, et al. Bioaccessibility of PAHs in contaminated soils: Comparison of five in vitro methods with Tenax as a sorption sink. Sci Total Environ. 2017;601602: 968-974.

41. Li C, Zhang R, Li Y, Zhang S, Gao P, Cui X, et al. Relative bioavailability and bioaccessibility of PCBs in soils based on a mouse model and Tenax-improved physiologically-based extraction test. Chemosphere. 2017;186: 709-715.

42. Bajaj P, Chowdhury SK, Yucha R, Kelly EJ, Xiao G. Emerging Kidney Models to Investigate Metabolism, Transport, and Toxicity of Drugs and Xenobiotics. Drug Metab Dispos. 2018;46: 16921702.

43. Shibutani ST, Saitoh T, Nowag H, Münz C, Yoshimori T. Autophagy and autophagy-related proteins in the immune system. Nature Immunology. 2015. pp. 1014-1024. doi:10.1038/ni.3273

44. Peters A, Nawrot TS, Baccarelli AA. Hallmarks of environmental insults. Cell. 2021. doi:10.1016/j.cell.2021.01.043

45. Orešič M, McGlinchey A, Wheelock CE, Hyötyläinen T. Metabolic Signatures of the ExposomeQuantifying the Impact of Exposure to Environmental Chemicals on Human Health. Metabolites. 2020;10. doi:10.3390/metabo10110454

46. Schaue D, Kachikwu EL, McBride WH. Cytokines in radiobiological responses: a review. Radiat Res. 2012;178: 505-523. 
47. Chhetri DR, Chhetri A, Shahi N, Tiwari S, Karna SKL, Lama D, et al. Isaria tenuipes Peck, an entomopathogenic fungus from Darjeeling Himalaya: Evaluation of in-vitro antiproliferative and antioxidant potential of its mycelium extract. BMC Complement Med Ther. 2020;20: 185.

48. Barros MB de L, de Almeida Paes R, Schubach AO. Sporothrix schenckii and Sporotrichosis. Clin Microbiol Rev. 2011;24: 633-654.

49. Danella Figo D, De Amicis K, Neiva Santos de Aquino D, Pomiecinski F, Gadermaier G, Briza P, et al. Cashew Tree Pollen: An Unknown Source of IgE-Reactive Molecules. Int J Mol Sci. 2019;20. doi:10.3390/ijms20102397

50. Xu M, Gao P, Yang Z, Su L, Wu J, Yang G, et al. Biochar impacts on phosphorus cycling in rice ecosystem. Chemosphere. 2019;225: 311-319.

51. Gao P, Liu Y, Wang Y, Liu X, Wang Z, Ma LQ. Spatial and temporal changes of P and Ca distribution and fractionation in soil and sediment in a karst farmland-wetland system. Chemosphere. 2019;220: 644-650.

52. Xu M, Barbosa da Silva E, Gao P, Liao R, Wu J, Ma J, et al. Biochar impact on chromium accumulation by rice through Fe microbial-induced redox transformation. J Hazard Mater. 2020;388: 121807.

53. da Silva EB, Gao P, Xu M, Guan D, Tang X, Ma LQ. Background concentrations of trace metals As, $\mathrm{Ba}, \mathrm{Cd}, \mathrm{Co}, \mathrm{Cu}, \mathrm{Ni}, \mathrm{Pb}, \mathrm{Se}$, and $\mathrm{Zn}$ in 214 Florida urban soils: Different cities and land uses. Environ Pollut. 2020;264: 114737.

54. Jiang C, Zhang X, Gao P, Chen Q, Snyder M. Decoding personal biotic and abiotic airborne exposome. Nat Protoc. 2021. doi:10.1038/s41596-020-00451-8

55. Barupal DK, Fiehn O. Generating the Blood Exposome Database Using a Comprehensive Text Mining and Database Fusion Approach. Environ Health Perspect. 2019;127: 97008.

56. Wishart D, Arndt D, Pon A, Sajed T, Guo AC, Djoumbou Y, et al. T3DB: the toxic exposome database. Nucleic Acids Res. 2015;43: D928-34.

57. Neveu V, Nicolas G, Salek RM, Wishart DS, Scalbert A. Exposome-Explorer 2.0: an update incorporating candidate dietary biomarkers and dietary associations with cancer risk. Nucleic Acids Res. 2020;48: D908-D912.

58. Shen X, Wang R, Xiong X, Yin Y, Cai Y, Ma Z, et al. Metabolic reaction network-based recursive metabolite annotation for untargeted metabolomics. Nat Commun. 2019;10: 1516.

59. Röst HL, Schmitt U, Aebersold R, Malmström L. pyOpenMS: a Python-based interface to the OpenMS mass-spectrometry algorithm library. Proteomics. 2014;14: 74-77.

60. Röst HL, Liu Y, D'Agostino G, Zanella M, Navarro P, Rosenberger G, et al. TRIC: an automated alignment strategy for reproducible protein quantification in targeted proteomics. Nat Methods. 2016;13: 777-783.

61. Contrepois K, Jiang L, Snyder M. Optimized Analytical Procedures for the Untargeted Metabolomic Profiling of Human Urine and Plasma by Combining Hydrophilic Interaction (HILIC) and ReversePhase Liquid Chromatography (RPLC)-Mass Spectrometry. Mol Cell Proteomics. 2015;14: 16841695. 
821 62. Dunn WB, Broadhurst D, Begley P, Zelena E, Francis-McIntyre S, Anderson N, et al. Procedures for large-scale metabolic profiling of serum and plasma using gas chromatography and liquid chromatography coupled to mass spectrometry. Nat Protoc. 2011;6: 1060-1083.

63. Li S, Park Y, Duraisingham S, Strobel FH, Khan N, Soltow QA, et al. Predicting network activity from high throughput metabolomics. PLoS Comput Biol. 2013;9: e1003123.

64. Pirhaji L, Milani P, Leidl M, Curran T, Avila-Pacheco J, Clish CB, et al. Revealing diseaseassociated pathways by network integration of untargeted metabolomics. Nat Methods. 2016;13: 770-776.

65. Yolum P, Güngör T, Gürgen F, Özturan C. Computer and Information Sciences - ISCIS 2005: 20th International Symposium, Istanbul, Turkey, October 26 -- 28, 2005, Proceedings. Springer Science \& Business Media; 2005. 


\section{Supplementary information}
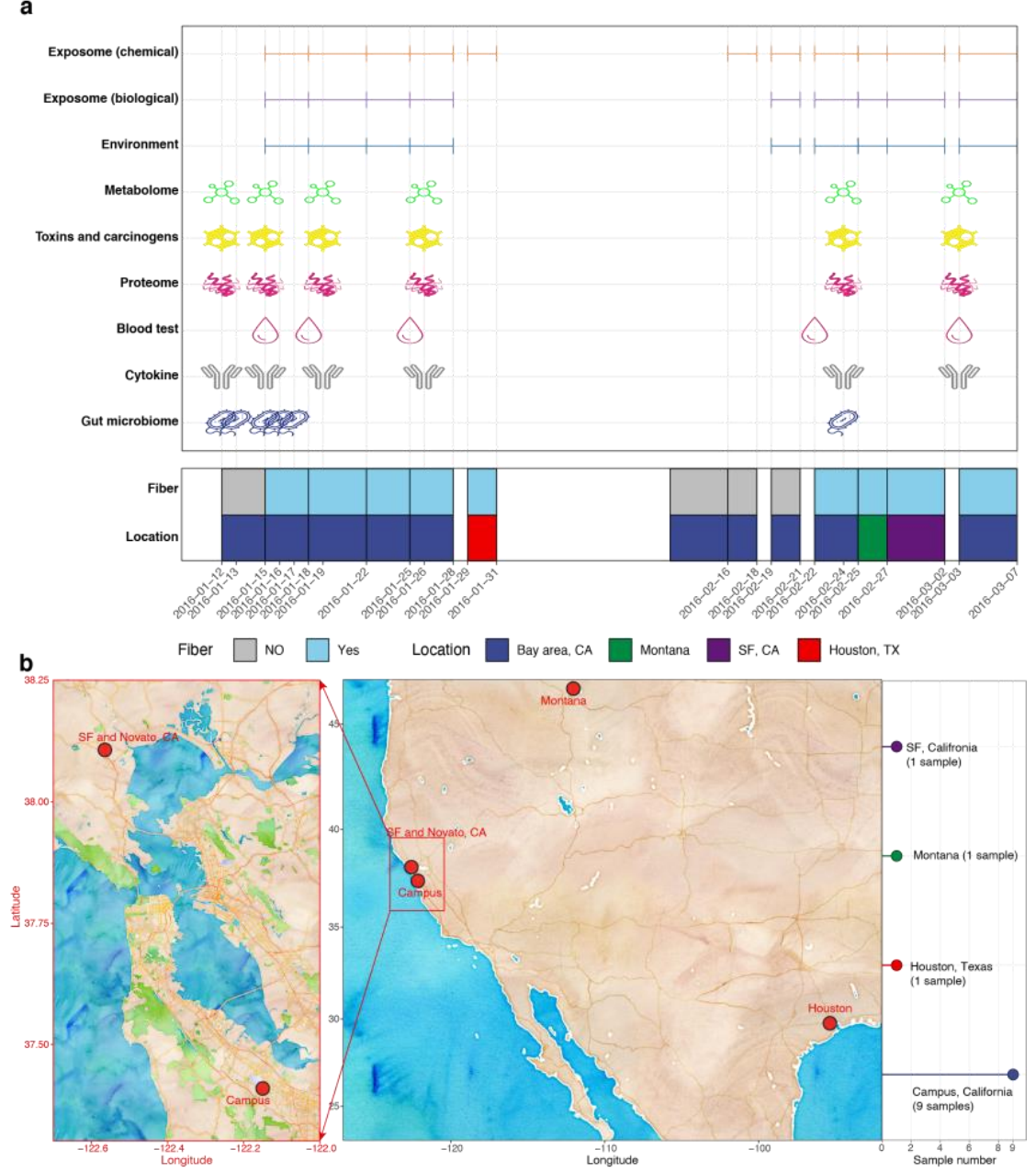

835 Figure S1. A detailed overview of sample collection. (a) Sample collection time points/periods

836 for all exposome and internal multi-omics datasets. Corresponding fiber intake and geographical

837 locations were also provided. (b) Collection locations for the exposome samples. 

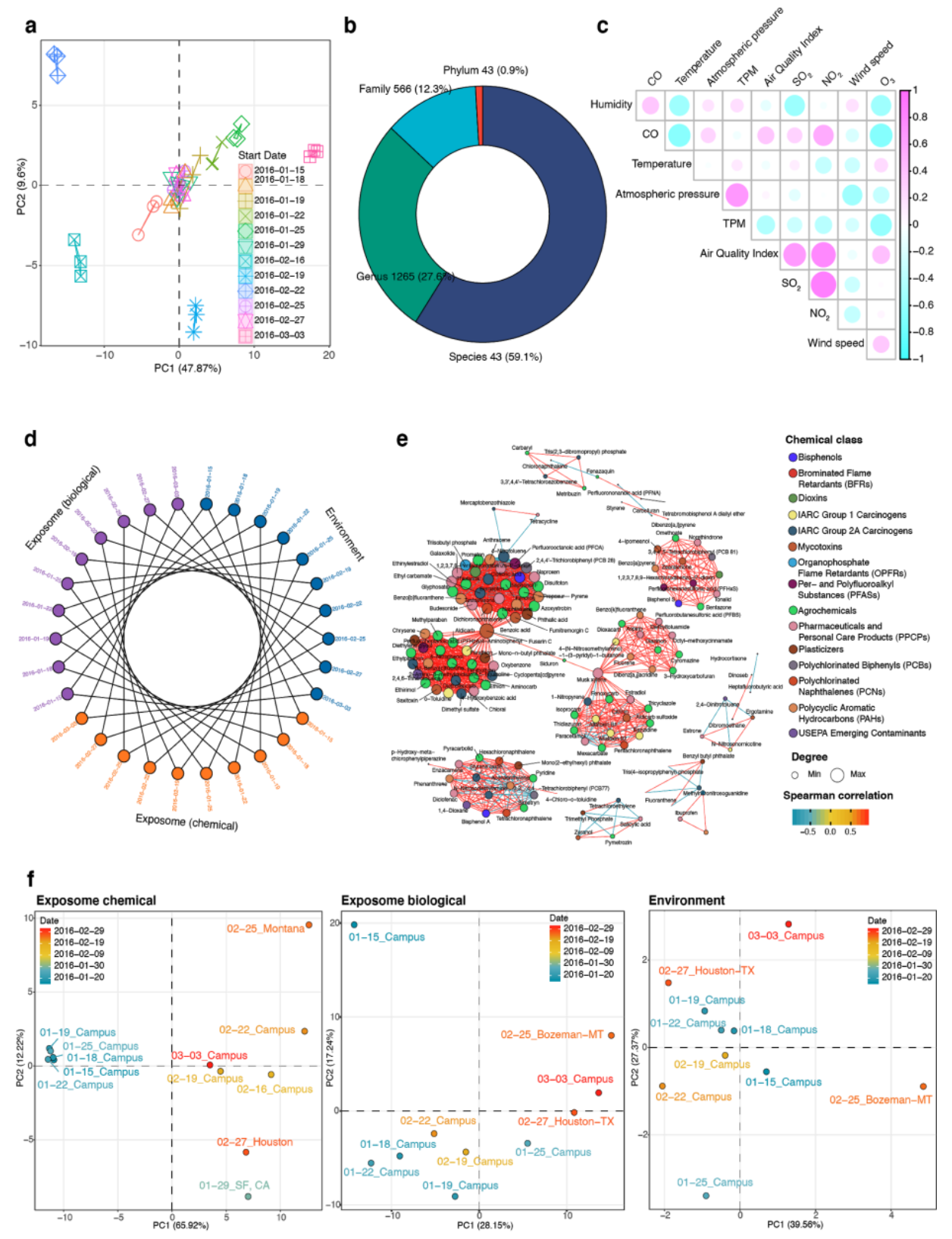

839 Figure S2. Overview of the exposome data. (a) PCA plot shows the data quality of chemical

840 exposome data. For each sample, 3 repeats were acquired. (b) The number and percentage of

841 biological exposome data at different taxonomic ranks. (c) Correlation plots between all

842 environmental factors. (d) Sample matching for 3 exposome domains. (e) Intra correlation

843 network for the chemical exposome. (f) PCA plots of the 3 exposome domains based on the

844 sampling time and locations. 
a

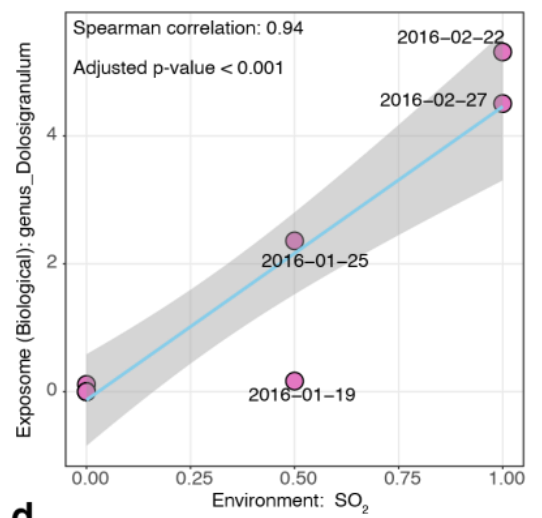

d

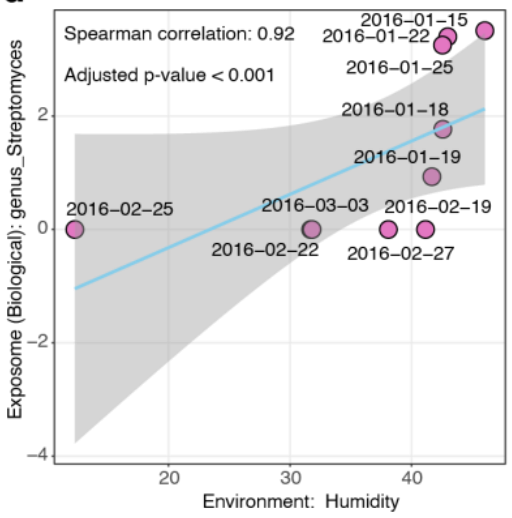

b

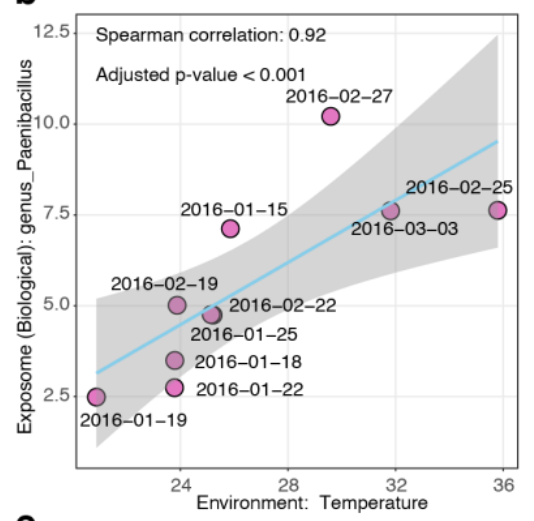

e

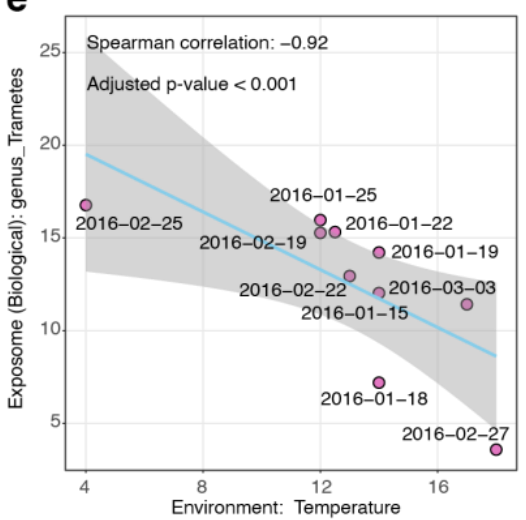

\section{C}

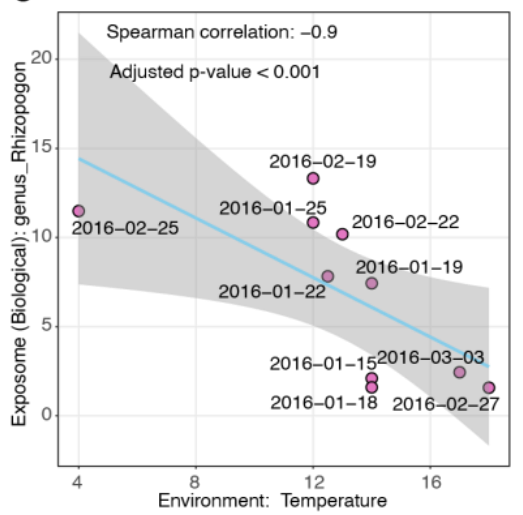

846 Figure S3. Representative Spearman correlation plots between environment factors and

847 microbes. 

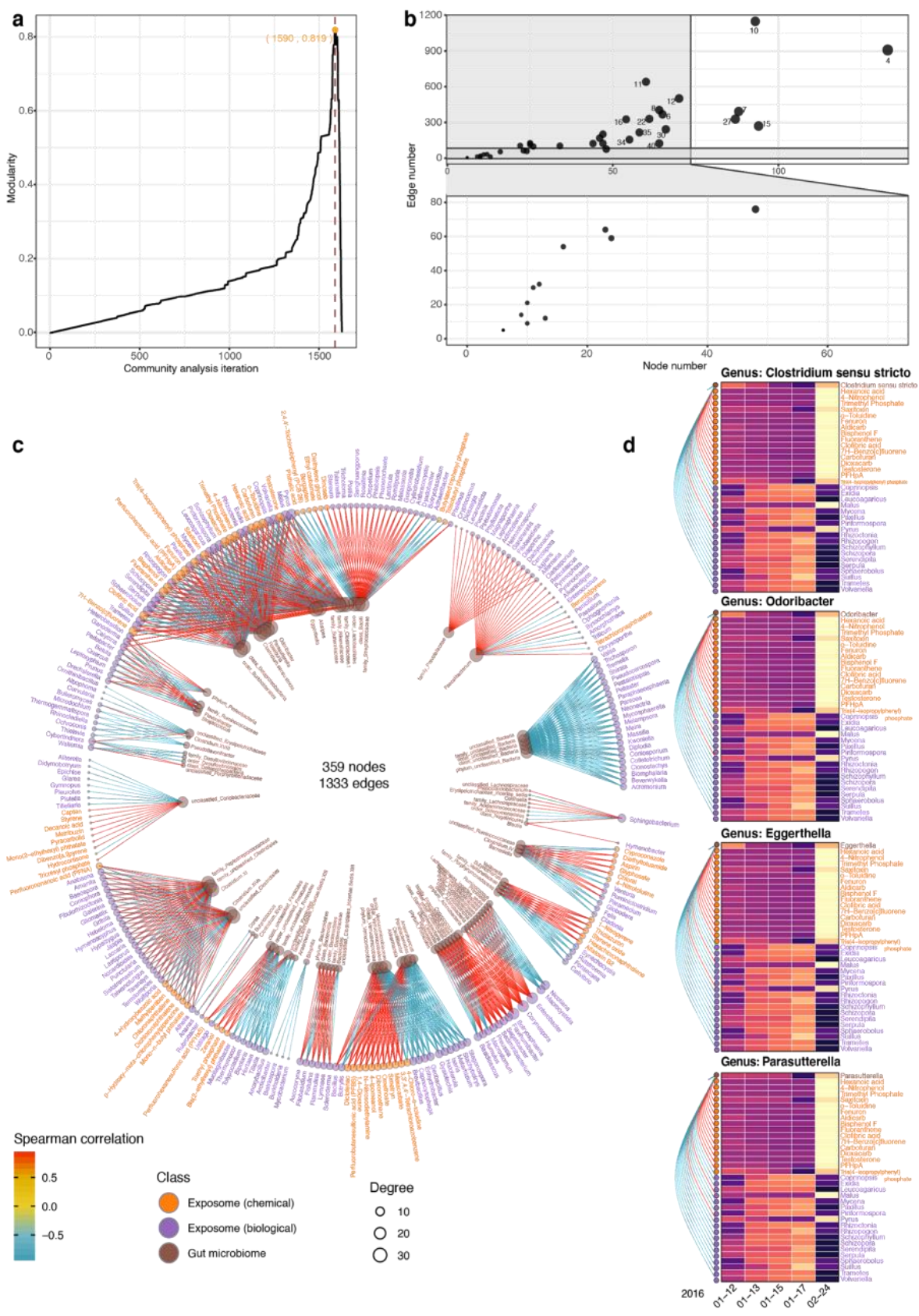

849 Figure $\mathbf{S 4}$. The exposome and internal multi-omics analyses. (a) The maximum modularity in

850 the exposome and internal-omes correlation network community analysis was 0.819 . (b) Node

851 and edge numbers for all the subnetworks in the community analysis. (c) The complete

852 correlation network between the exposome and the gut microbiome $(|\mathrm{r}|>0.9$; q-value $<0.05)$.

853 (d) Representative personal gut bacteria that has 34 significant correlations with the exposome 854 components $(|\mathrm{r}|>0.9 ; \mathrm{q}$-value $<0.05)$. 


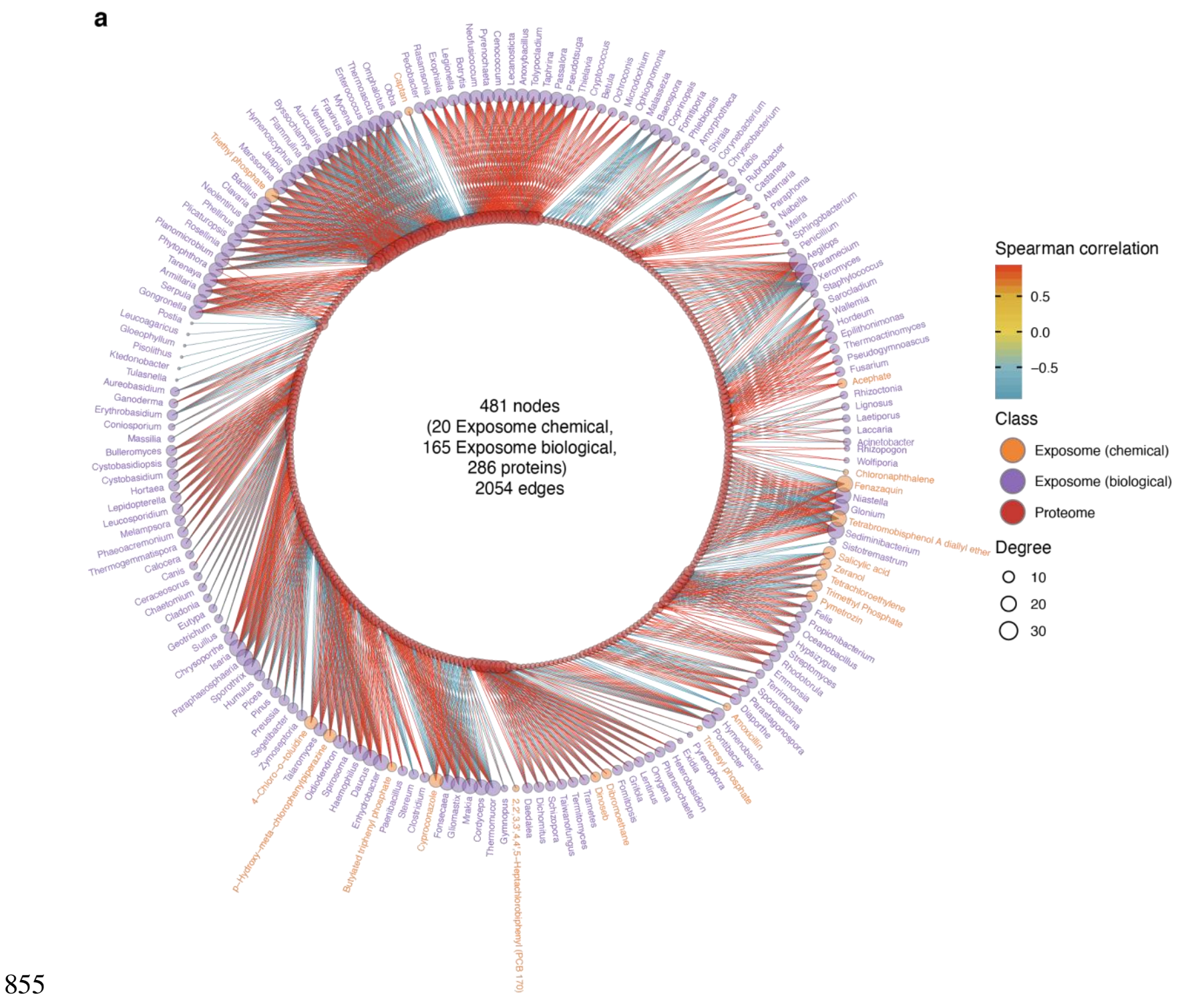

856 Figure S5. The complete correlation network between the exposome and proteome $(|\mathrm{r}|>0.9$; q-

857 value $<0.05)$. 
a

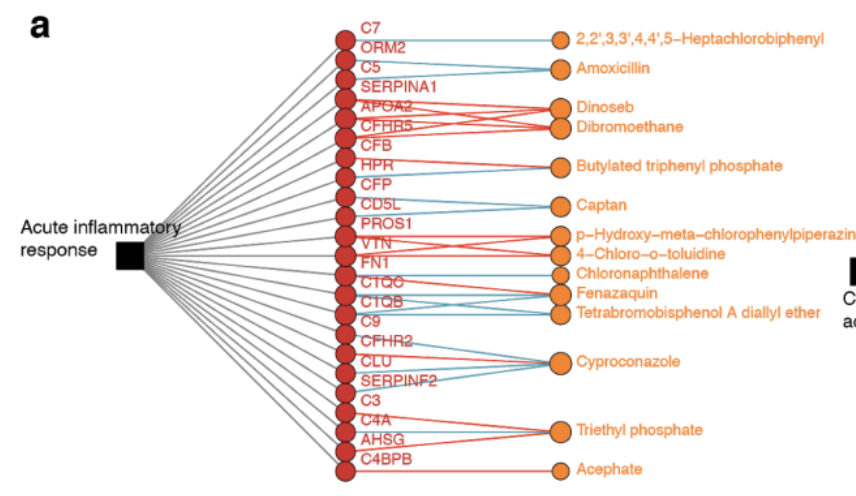

C

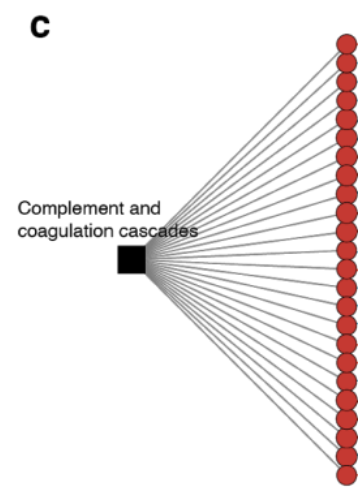

b
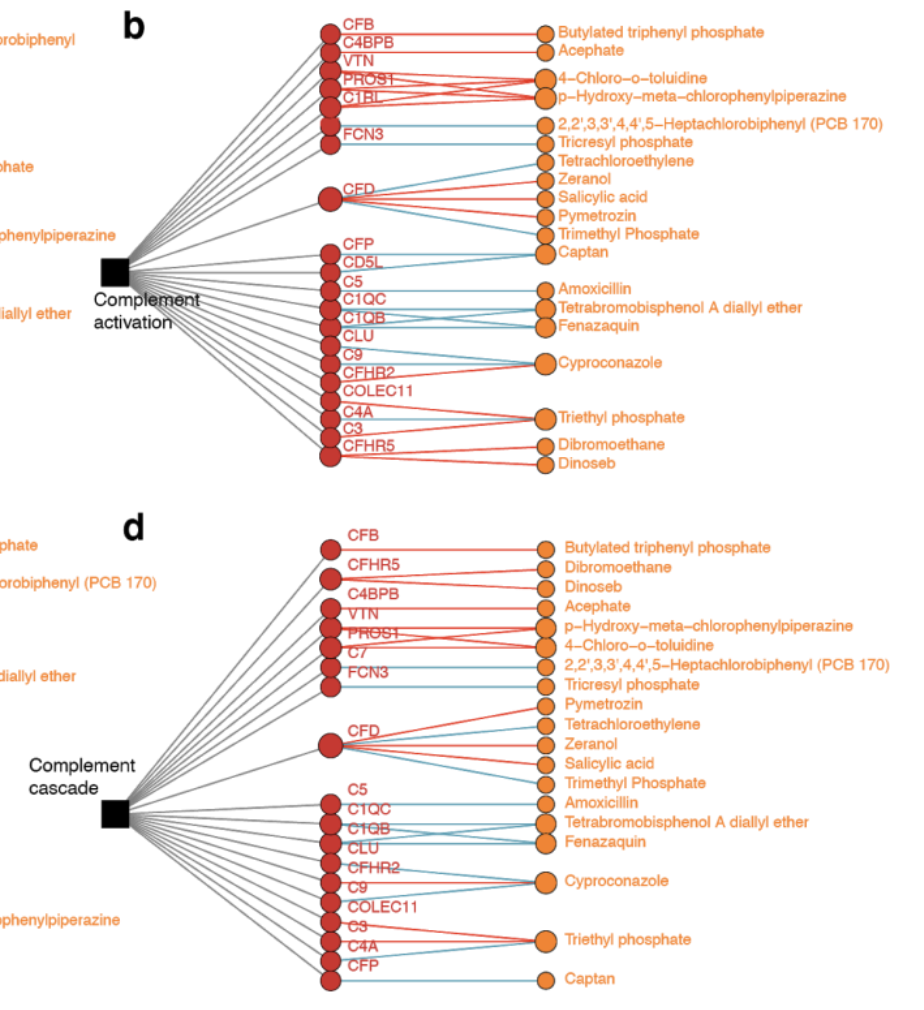

\section{e}
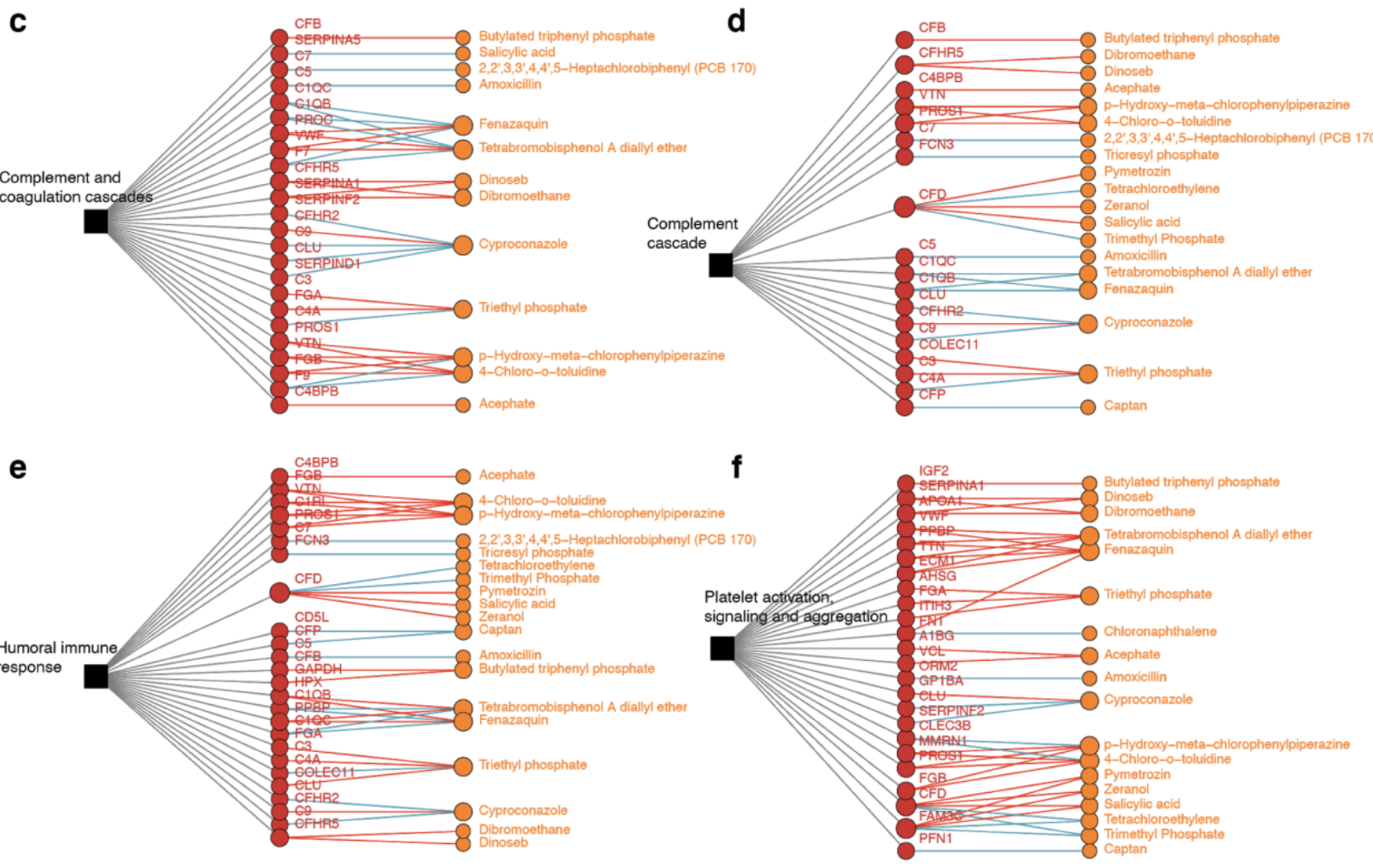

h

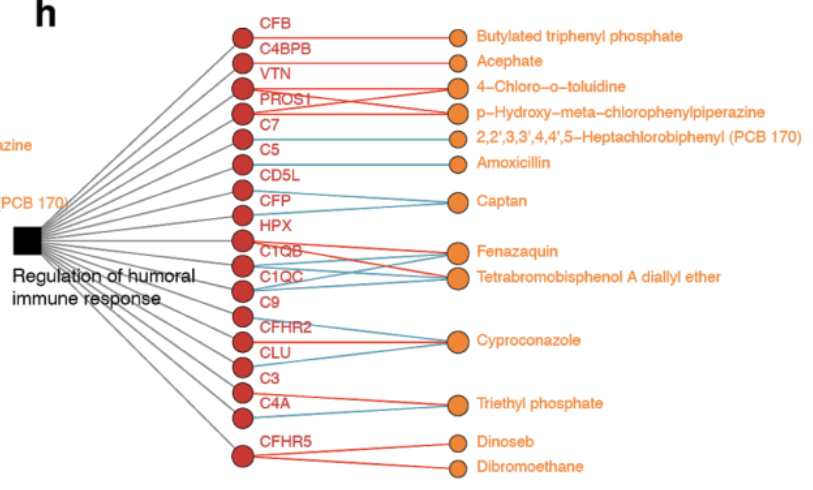

g

CFB $\quad$ Butylated triphenyl phosphate

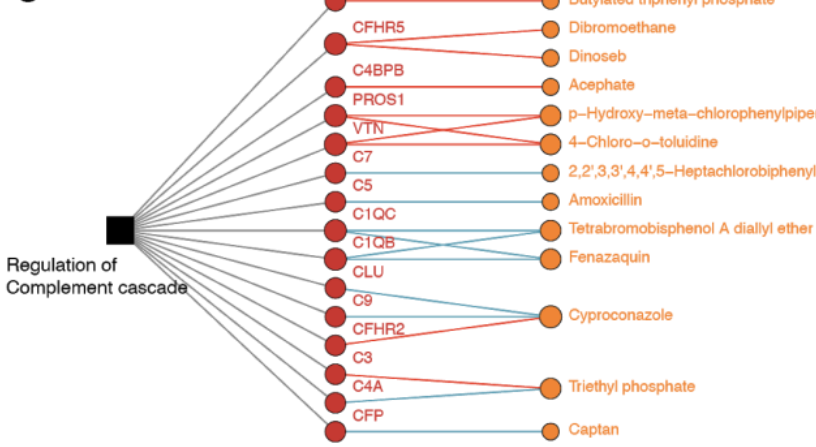

859 Figure S6. The detailed networks between the exposome and proteins for each signaling

860 pathway $(|\mathrm{r}|>0.9 ; \mathrm{q}-\mathrm{value}<0.05)$ 


\section{a}

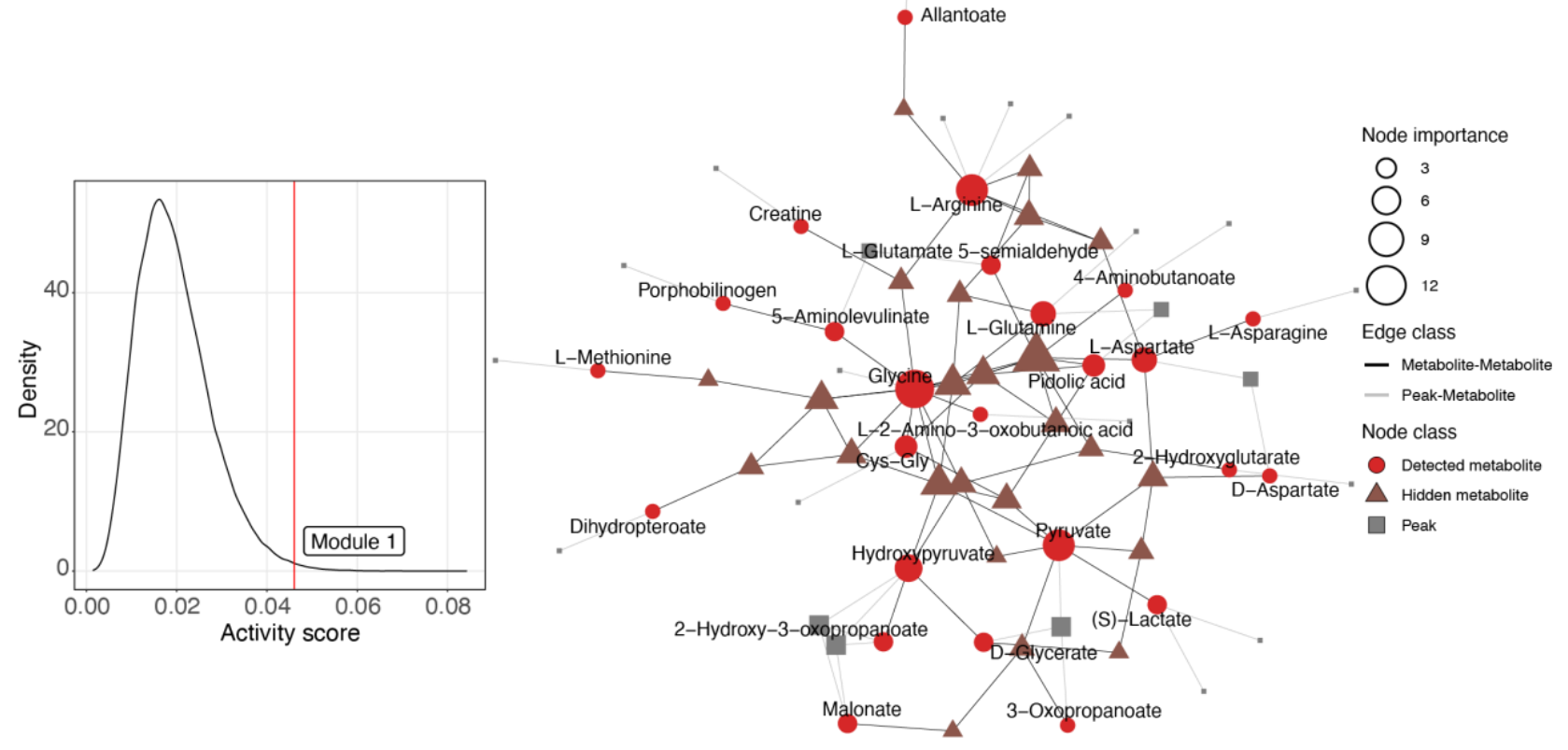

b
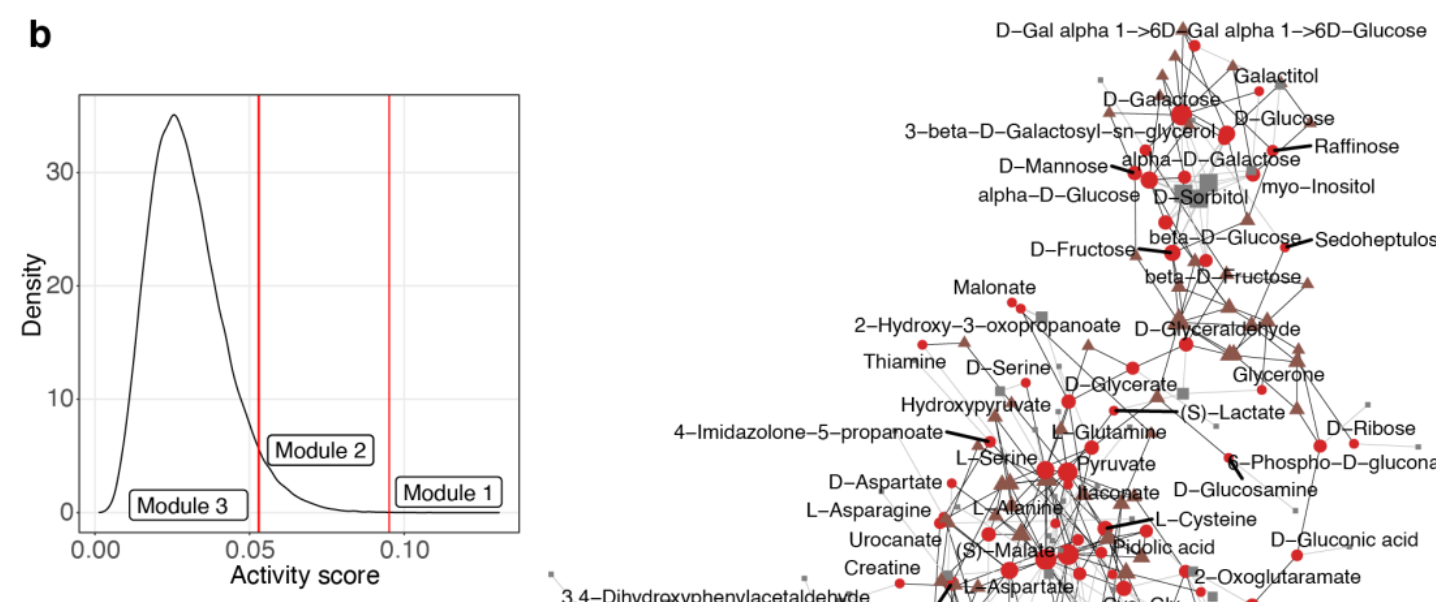

3-beta-D-Glucase

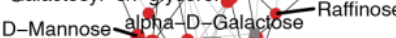

alpha-D-Glucose D-Sorbitol/myo-Inositol

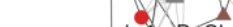

D-Fructose bett-D-Glucose-Sedoheptulose Edge class
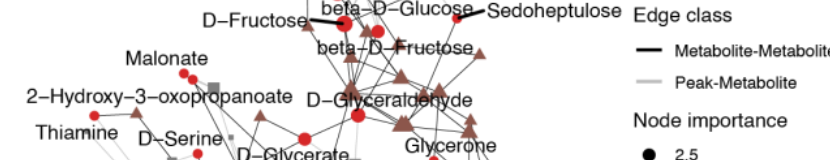

Hydroxypyrtivate,

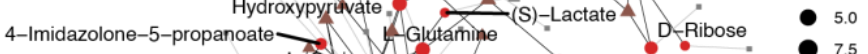

LAsperine fyruvate Phospho-D-gluconate 7.5

Asparagine Alaning idasonate D-Glucosamine

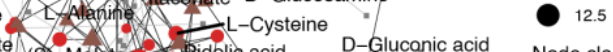

Creatine ST-Máate X pidolic acid

3-Dihydroxyphenylacetaldehyde Detected metabolite

3,4-Dihydroxymandelates-Methoxytyramine L-Citrulline Citrate Gys-G D-Glucono-1,5-lactone $\mathbf{\Delta}$ Hidden metabolite

3-Methoxy-4-hydroxymandelate L-Noradrenaline Isocitrate, = Peak

3,4-Dihydroxymatruelaldehyde Allantoate

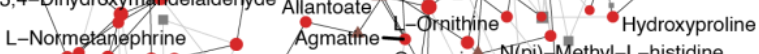

L-Adrenoline Carnosine N(pi)-Methyl-L-histidine

(-) Urphobilinogen

$\mathrm{N}-$ Acetylornithine

862 Figure S7. Feature-based network analysis for metabolic features significantly correlated with

863 the exposome (Methods). (a) Chemical exposome. Left panel, the null distribution of activity

864 scores and only module 1 was significant. Right panel, network constructed by significant

865 modules. (b) Biological exposome. Left panel, the null distribution of activity scores and modules 1, 2 and 3 were significant. Right panel, network constructed by significant modules. 
bioRxiv preprint doi: https://doi.org/10.1101/2021.05.05.442855; this version posted August 13, 2021. The copyright holder for this preprint (which was not certified by peer review) is the author/funder. All rights reserved. No reuse allowed without permission.

a

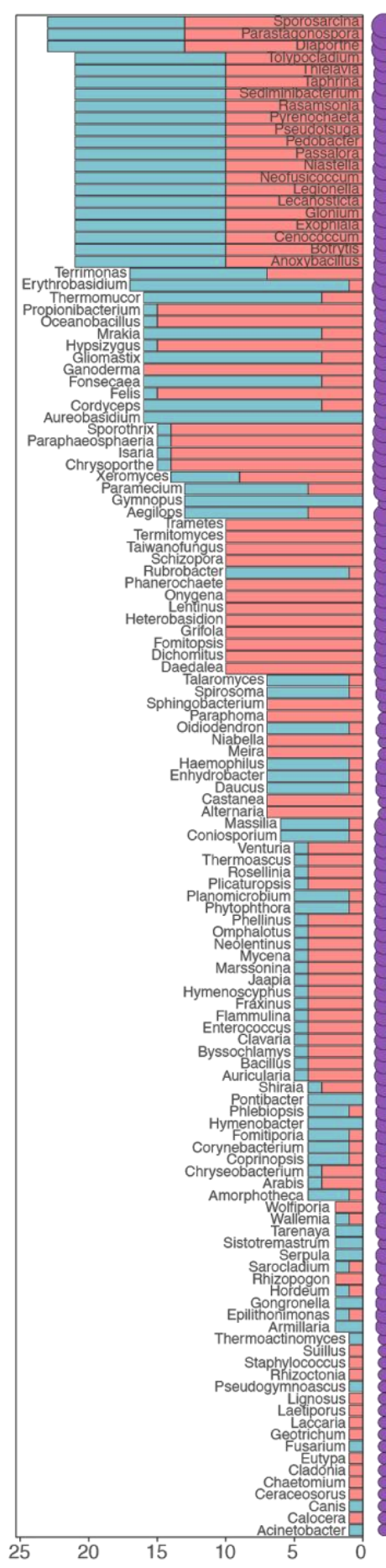

Figure S8. The complete network between the exposome and metabolite for each metabolic
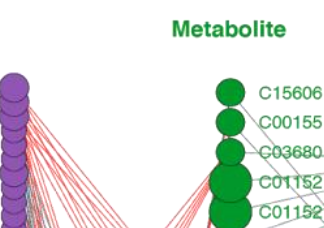

C00155

$\left\{\begin{array}{l}0001560 \\ 001152\end{array}\right.$

001152 Co1044

C 900785

C00437

- 000327

- 005665

100386

. $\begin{aligned} & c 00149 \\ & 000345\end{aligned}$

8 o00257

coorig:

1 cow

1069776

60850

$\left\{\begin{array}{l}00038 \\ 00668\end{array}\right.$

000300
005584

3005624

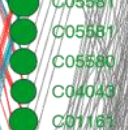

13 colforit (1)

-

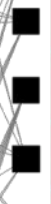

Neuroactive ligand-receptor interaction

beta-Alanine metabolism

D CAMP signaling pattway

a

Glycolysis / Gluconeogenøsis

Fructose and mannose metabolism

1 Arginine biosynthesis

- Citrate cycle (TCA cycle)

Glutathione metabolism

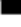

Pantothenate ard CoA biosynthesis

-

Taurine and hypotaurine metabolism

1

Synaptic vesicle cycle
C15606

Galactose metabolism
0
Pathway number

25

Compound number

pathway $(|\mathrm{r}|>0.9$; q-value $<0.05)$. 


\section{Supplementary Note}

\section{Note 1. The session information in the study}

873 R version $4.0 .3(2020-10-10)$

874 Platform: x86_64-apple-darwin17.0 (64-bit)

875 Running under: macOS Big Sur 10.16

876

877 Matrix products: default

878 LAPACK: /Library/Frameworks/R.framework/Versions/4.0/Resources/lib/libRlapack.dylib

880 locale:

881

[1] en_US.UTF-8/en_US.UTF-8/en_US.UTF-8/C/en_US.UTF-8/en_US.UTF-8

882

883 attached base packages:

884 [1] parallel stats4 grid stats graphics grDevices utils datasets methods

885 [10] base

886

887 other attached packages:

888 [1] ReactomePA_1.34.0 org.Hs.eg.db_3.10.0 AnnotationDbi_1.52.0 IRanges_2.24.1

889 [5] S4Vectors_0.28.1 Biobase_2.50.0 BiocGenerics_0.36.0 clusterProfiler_3.18.0

890 [9] circlize_0.4.11 ComplexHeatmap_2.6.2 patchwork_1.1.1 plsVarSel_0.9.6

891 [13] pls_2.7-3 openxlsx_4.2.3 plyr_1.8.6 ggalluvial_0.12.3

892 [17] tidygraph_1.2.0 ggraph_2.0.4 igraph_1.2.6 forcats_0.5.0 
$\begin{array}{llccc}893 & \text { [21] stringr_1.4.0 } & \text { dplyr_1.0.2 } & \text { purrr_0.3.4 } & \text { readr_1.4.0 } \\ 894 & \text { [25] tidyr_1.1.2 } & \text { tibble_3.0.4 } & \text { ggplot2_3.3.3 } & \text { tidyverse_1.3.0 }\end{array}$

895

896 loaded via a namespace (and not attached):

$897 \quad$ [1] readxl_1.3.1 shadowtext_0.0.7 backports_1.2.1

898 [4] fastmatch_1.1-0 sxtTools_0.99.01 splines_4.0.3

899 [7] BiocParallel_1.24.1 crosstalk_1.1.0.1 digest_0.6.27

$900 \quad[10]$ htmltools_0.5.0 GOSemSim_2.16.1 rsconnect_0.8.16

901 [13] magick_2.5.2 viridis_0.5.1 GO.db_3.10.0

902 [16] wesanderson_0.3.6 fansi_0.4.1 magrittr_2.0.1

903 [19] checkmate_2.0.0 memoise_1.1.0 cluster_2.1.0

904 [22] graphlayouts_0.7.1 modelr_0.1.8 matrixStats_0.57.0

905 [25] bdsmatrix_1.3-4 prettyunits_1.1.1 enrichplot_1.10.1

906 [28] colorspace_2.0-0 blob_1.2.1 rvest_0.3.6

907 [31] rappdirs_0.3.1 ggrepel_0.9.0 xfun_0.19

908 [34] haven_2.3.1 crayon_1.3.4 jsonlite_1.7.2

909 [37] graph_1.68.0 scatterpie_0.1.5 glue_1.4.2

910 [40] polyclip_1.10-0 gtable_0.3.0 webshot_0.5.2

911 [43] genalg_0.2.0 GetoptLong_1.0.5 graphite_1.36.0

912 [46] shape_1.4.5 scales_1.1.1 DOSE_3.16.0

913 [49] mvtnorm_1.1-1 DBI_1.1.0 miniUI_0.1.1.1

914 [52] Rcpp_1.0.5.4 progress_1.2.2 xtable_1.8-4

915 [55] viridisLite_0.3.0 clue_0.3-58 gridGraphics_0.5-1 
916 [58] bit_4.0.4 reactome.db_1.70.0 htmlwidgets 1.5 .3

917 [61] httr_1.4.2 fgsea_1.16.0 RColorBrewer_1.1-2

918 [64] ellipsis_0.3.1 pkgconfig_2.0.3 farver_2.0.3

919 [67] dbplyr_2.0.0 utf8_1.1.4 manipulateWidget_0.10.1

920 [70] later_1.1.0.1 ggplotify_0.0.5 tidyselect_1.1.0

921 [73] labeling_0.4.2 rlang_0.4.10 reshape2_1.4.4

922 [76] munsell_0.5.0 cellranger_1.1.0 tools_4.0.3

923 [79] downloader_0.4 cli_2.2.0 generics_0.1.0

924 [82] RSQLite_2.2.1 broom_0.7.3 evaluate_0.14

925 [85] fastmap_1.0.1 yaml_2.2.1 knitr_1.30

926 [88] bit64_4.0.5 fs_1.5.0 $\quad$ zip_2.1.1

927 [91] rgl_0.103.5 mime_0.9 praznik_8.0.0

928 [94] DO.db_2.9 xml2_1.3.2 compiler_4.0.3

929 [97] rstudioapi_0.13 MSQC_1.0.2 png_0.1-7

930 [100] reprex_0.3.0 tweenr_1.0.1 stringi_1.5.3

931 [103] lattice_0.20-41 Matrix_1.2-18 ggsci_2.9

932 [106] vctrs_0.3.6 pillar_1.4.7 lifecycle_0.2.0

933 [109] BiocManager_1.30.10 GlobalOptions_0.1.2 data.table_1.13.6

934 [112] cowplot_1.1.1 httpuv_1.5.4 qvalue_2.22.0

935 [115] R6_2.5.0 promises_1.1.1 gridExtra_2.3

936 [118] sessioninfo_1.1.1 MASS_7.3-53 assertthat_0.2.1

937 [121] rjson_0.2.20 withr_2.3.0 $\quad$ hms_0.5.3

938 [124] rmarkdown_2.6 rvcheck_0.1.8 Cairo_1.5-12.2 
[127] ggnewscale_0.4.4 ggforce_0.3.2 shiny_1.5.0

940 [130] lubridate_1.7.9.2

942 Note 2. The food log (fiber intake) of the participant in this study

943 From 1/15/2016 to 1/31/2016, the participant took 20 grams of arabinoxylan daily. From 2/22/2016

944 to $3 / 17 / 2016$, the participant took 10 grams of guar gum daily.

945

946 Supplementary Table 1. Adduct list for metabolite annotation in this study.

\begin{tabular}{|c|c|}
\hline Mode & Adduct \\
\hline RPLC positive mode & $\begin{array}{l}(\mathrm{M}+\mathrm{H})^{+},\left(\mathrm{M}+\mathrm{H}-\mathrm{H}_{2} \mathrm{O}\right)^{+},\left(\mathrm{M}+\mathrm{H}-2 \mathrm{H}_{2} \mathrm{O}\right)^{+}, \\
\left(\mathrm{M}+\mathrm{NH}_{4}\right)^{+},(\mathrm{M}+\mathrm{Na})^{+},(\mathrm{M}-\mathrm{H}+2 \mathrm{Na})^{+},(\mathrm{M}- \\
2 \mathrm{H}+3 \mathrm{Na})^{+},(\mathrm{M}+\mathrm{K})^{+},(\mathrm{M}-\mathrm{H}+2 \mathrm{~K})^{+},(\mathrm{M}- \\
2 \mathrm{H}+3 \mathrm{~K})^{+},\left(\mathrm{M}+\mathrm{CH}{ }_{3} \mathrm{CN}+\mathrm{H}\right)^{+}, \\
\left(\mathrm{M}+\mathrm{CH}_{3} \mathrm{CN}+\mathrm{Na}\right)^{+},(2 \mathrm{M}+\mathrm{H})^{+},\left(2 \mathrm{M}+\mathrm{NH}_{4}\right)^{+}, \\
(2 \mathrm{M}+\mathrm{Na})^{+},(2 \mathrm{M}+\mathrm{K})^{+},(\mathrm{M}+\mathrm{HCOO}+2 \mathrm{H})^{+}\end{array}$ \\
\hline RPLC negative mode & $\begin{array}{l}(\mathrm{M}-\mathrm{H})^{-},\left(\mathrm{M}-\mathrm{H}_{2} \mathrm{O}-\mathrm{H}\right)^{-},(\mathrm{M}+\mathrm{Na}-2 \mathrm{H})^{-},(\mathrm{M}+\mathrm{K}- \\
2 \mathrm{H})^{-},\left(\mathrm{M}+\mathrm{NH}_{4}-2 \mathrm{H}\right)^{-},(2 \mathrm{M}-\mathrm{H})^{-},(\mathrm{M}+\mathrm{F})^{-}\end{array}$ \\
\hline HILIC positive mode & $\begin{array}{l}(\mathrm{M}+\mathrm{H})^{+},\left(\mathrm{M}+\mathrm{H}-\mathrm{H}_{2} \mathrm{O}\right)^{+},\left(\mathrm{M}+\mathrm{H}-2 \mathrm{H}_{2} \mathrm{O}\right)^{+}, \\
\left(\mathrm{M}+\mathrm{NH}_{4}\right)^{+},(\mathrm{M}+\mathrm{Na})^{+},(\mathrm{M}-\mathrm{H}+2 \mathrm{Na})^{+},(\mathrm{M}- \\
2 \mathrm{H}+3 \mathrm{Na})^{+},(\mathrm{M}+\mathrm{K})^{+},(\mathrm{M}-\mathrm{H}+2 \mathrm{~K})^{+},(\mathrm{M}- \\
2 \mathrm{H}+3 \mathrm{~K})^{+},\left(\mathrm{M}+\mathrm{CH}{ }_{3} \mathrm{CN}+\mathrm{H}\right)^{+}\end{array}$ \\
\hline
\end{tabular}




\begin{tabular}{|l|l|}
\hline & $\begin{array}{l}\left(\mathrm{M}+\mathrm{CH}_{3} \mathrm{CN}+\mathrm{Na}\right)^{+},(2 \mathrm{M}+\mathrm{H})^{+},(2 \mathrm{M}+\mathrm{NH})^{+}, \\
(2 \mathrm{M}+\mathrm{Na})^{+},(2 \mathrm{M}+\mathrm{K})^{+},(\mathrm{M}+\mathrm{HCOO}+2 \mathrm{H})^{+}\end{array}$ \\
\hline HILIC negative mode & $\begin{array}{l}(\mathrm{M}-\mathrm{H})^{-},\left(\mathrm{M}-\mathrm{H}_{2} \mathrm{O}-\mathrm{H}\right)^{-},(\mathrm{M}+\mathrm{Na}-2 \mathrm{H})^{-},(\mathrm{M}+\mathrm{K}- \\
2 \mathrm{H})^{-},\left(\mathrm{M}+\mathrm{NH}_{4}-2 \mathrm{H}\right)^{-},(2 \mathrm{M}-\mathrm{H})^{-},\left(\mathrm{M}+\mathrm{CH}_{3} \mathrm{COO}\right)^{-}\end{array}$ \\
\hline
\end{tabular}

948 Supplementary Data

949 Supplementary Data 1. All exposome and internal multi-omics data used in this study.

950 Supplementary Data 2. The detailed information of the intra-correlation network of the

951 chemical exposome.

952 Supplementary Data 3. The detailed information of the personal exposome cloud.

953 Supplementary Data 4. The detailed information of the inter-correlation network of the

954 exposome and internal-multi-omics data.

955 Supplementary Data 5. Pathway enrichment results for proteins connected with the chemical

956 exposome.

957 Supplementary Data 6. Pathway enrichment results for proteins connected with the biological

958 exposome.

959 Supplementary Data 7. Pathway enrichment results for metabolic peaks connected with the

960 chemical exposome.

961 Supplementary Data 8. Pathway enrichment results for metabolic peaks connected with the

962 biological exposome.

963 Supplementary Data 9. The comparison between metabolite annotations from traditional

964 methods and metabolic feature-based network analysis.

965 Supplementary Data 10. The contributions of the exposome on blood tests and cytokines. 